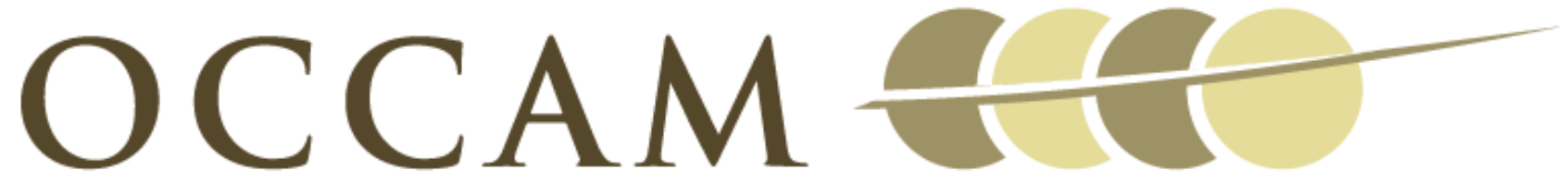

OXFORD CENTRE FOR COLLABORATIVE APPLIED MATHEMATICS

\author{
Report Number 10/50
}

A model for the anisotropic response of fibrous soft tissues using six discrete fibre bundles

by

Cormac Flynn, MB Rubin, Poul Nielsen

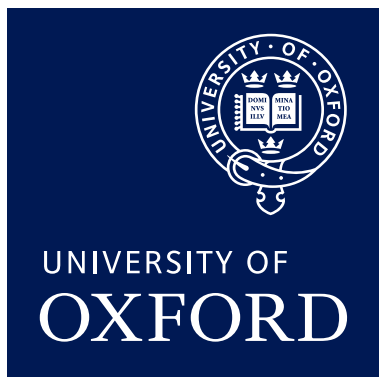

Oxford Centre for Collaborative Applied Mathematics Mathematical Institute 24 - 29 St Giles'

Oxford

OX1 3LB

England 



\section{Full title: A model for the anisotropic response of fibrous soft tissues using six discrete fibre bundles}

\section{Short title: A model for fibrous soft tissues using discrete fibre bundles}

Corresponding Author:

Cormac Flynn,

Auckland Bioengineering Institute,

University of Auckland,

70 Symonds Street,

Auckland,

New Zealand.

Tel.: $\quad+6493737599$ ext 83010

Fax: $\quad+6493677157$

c.flynn@auckland.ac.nz

www.abi.auckland.ac.nz

MB Rubin

mbrubin@tx.technion.ac.il

Poul Nielsen

p.nielsen@auckland.ac.nz

Word Count: 4300

\section{Sponsors}

This work was in part supported by the New Zealand Foundation for Research, Science and Technology, through grants NERF 139400 and NERF 9077/3608892. This publication is also based on work supported in part by Award No KUK-C1-01304, made by King Abdullah University of Science and Technology (KAUST).

\section{Keywords:}

Constitutive model; Soft tissue mechanics; Anisotropy; Analytical distribution functions; 


\section{Abstract}

The development of accurate constitutive models of fibrous soft-tissues is a challenging problem. Many consider the tissue to be a collection of fibres with a continuous distribution function representing their orientations. A novel discrete fibre model is presented consisting of six weighted fibre bundles. Each bundle is oriented such that they pass through opposing vertices of a regular icosahedron. A novel aspect of the model is the use of simple analytical distribution functions to simulate the undulated collagen fibres. This approach yields a closed form analytical expression for the strain energy function for the collagen fibre bundle that avoids the sometimes costly numerical integration of some statistical distribution functions. The elastin fibres are characterized by a neo-Hookean strain energy function. The model accurately simulates the biaxial stretching of rabbit-skin (error-of-fit 8.7\%), the uniaxial stretching of pig-skin (error-of-fit 7.6\%), equibiaxial loading of aortic valve cusp (error-of-fit $0.8 \%$ ), and the simple shear of rat septal myocardium (error-of-fit 9.1\%). The proposed model compares favourably with previously published softtissue models and alternative methods of representing undulated collagen fibres. The stiffness of collagen fibres predicted by the model ranges from $8.0 \mathrm{MPa}$ to $0.93 \mathrm{GPa}$. The stiffness of elastin fibres ranges from $2.5 \mathrm{kPa}$ to $154.4 \mathrm{kPa}$. The anisotropy of model resulting from the representation of the fibre field with a discrete number of fibres is also explored.

\section{Introduction}

Soft biological tissues are complex materials, which exhibit non-linear stressstrain behaviour, anisotropy, and viscoelasticity [1-3]. In the last few decades, significant effort has been devoted to the challenging problem of developing constitutive models that simulate soft tissue mechanical behaviour with sufficient accuracy [4-7]. The benefits of accurate constitutive models include improved techniques and devices to treat coronary disease [8], superior surgical incision methods [9], and better personal care products such as razors and sticky-plasters [10, $11]$.

The non-linear stress-strain relationship exhibited by many soft tissues is mainly due to the uncrimping of undulated collagen fibres within the tissue upon stretching 
[12]. Several constitutive models represent this uncrimping phenomenologically using exponential strain energy functions [13-16]. While phenomenological approaches can be useful, their application can be limited to specific deformations and their material parameters can lack physical meaning [17]. Physically-based methods model the uncrimping and engagement of collagen fibres using a normal distribution function [5], a Lorentz function [18], or a log-logistic distribution function [17]. While including these types of functions in constitutive models is straightforward, the evaluation of their integrals to obtain the stress-strain relationship can be cumbersome and computationally costly $[19,20]$. This becomes an obstacle when such constitutive models are used to solve complex boundary problems within a finite element framework [21]. Therefore, there is a need to derive constitutive models that do not require the numerical integration of distribution functions.

The anisotropic characteristics of soft tissues arise due to the preferred orientation and distribution of the collagen fibres [7, 12, 22]. Some previous constitutive models assume soft tissues to have families of collagen fibres, each of which are perfectly aligned with no dispersion $[17,18,23]$. These simplifying assumptions can give problems when predicting the mechanical response normal to the fibre directions [21]. Other soft tissue models use continuous distribution functions to represent the orientation of the fibres within the soft tissue $[5,7,16,21,24]$. Such models typically use a normal or von Mises distribution to represent the fibre population. As with the case for modelling collagen fibre undulation, integration of these functions can pose similar issues when the constitutive models are used in large finite element models.

This paper presents a constitutive model for fibrous soft tissues consisting of six discrete fibre bundles. The collagen fibre undulation is represented using distribution functions, which can be integrated to give analytical stress-strain expressions. The model is tested by evaluating its ability to simulate a variety of soft tissue deformation experiments.

\section{Constitutive model development}

\subsection{Icosahedron model of the fibre distribution}

From Elata and Rubin [25], it is possible to define six unit vectors $\mathbf{N}_{\mathbf{i}}$ parallel to the lines passing through opposing vertices of a regular icosahedron (Figure 1) by 


$$
\begin{gathered}
\mathbf{N}_{1}=\frac{2}{\sqrt{5}} \mathbf{e}_{1}+\frac{1}{\sqrt{5}} \mathbf{e}_{3} \\
\mathbf{N}_{2}=\frac{1}{2}\left(1-\frac{1}{\sqrt{5}}\right) \mathbf{e}_{1}+\sqrt{\frac{1}{2}\left(1+\frac{1}{\sqrt{5}}\right)} \mathbf{e}_{2}+\frac{1}{\sqrt{5}} \mathbf{e}_{3} \\
\mathbf{N}_{3}=-\frac{1}{2}\left(1+\frac{1}{\sqrt{5}}\right) \mathbf{e}_{1}+\sqrt{\frac{1}{2}\left(1-\frac{1}{\sqrt{5}}\right)} \mathbf{e}_{2}+\frac{1}{\sqrt{5}} \mathbf{e}_{3} \\
\mathbf{N}_{4}=-\frac{1}{2}\left(1+\frac{1}{\sqrt{5}}\right) \mathbf{e}_{1}-\sqrt{\frac{1}{2}\left(1-\frac{1}{\sqrt{5}}\right)} \mathbf{e}_{2}+\frac{1}{\sqrt{5}} \mathbf{e}_{3} \\
\mathbf{N}_{5}=\frac{1}{2}\left(1-\frac{1}{\sqrt{5}}\right) \mathbf{e}_{1}-\sqrt{\frac{1}{2}\left(1+\frac{1}{\sqrt{5}}\right)} \mathbf{e}_{2}+\frac{1}{\sqrt{5}} \mathbf{e}_{3} \\
\mathbf{N}_{6}=\mathbf{e}_{3}
\end{gathered}
$$

where $\mathbf{e}_{\mathbf{i}}(i=1,2,3)$ are fixed orthonormal base vectors of a rectangular Cartesian coordinate system and the vectors have been modified so that $\mathbf{N}_{\mathbf{i}}(i=1,2, \ldots 5)$ are equally distributed around the pole $\mathbf{N}_{\mathbf{6}}$ in the $\mathbf{e}_{\mathbf{3}}$ direction. These unit vectors $\mathbf{N}_{\mathbf{i}}$ are equally spaced on the surface of the sphere. It is also convenient to define six symmetric tensors $\mathbf{B}_{\mathbf{i}}$

$$
\mathbf{B}_{\mathbf{i}}=\mathbf{N}_{\mathbf{i}} \otimes \mathbf{N}_{\mathbf{i}}, \quad i=1,2 \ldots 6(\text { no sum on } i=1,2, \ldots 6)
$$

where $\otimes$ denotes the tensor product operator. Elata and Rubin [25] have shown that

$$
\sum_{i=1}^{6} \mathbf{B}_{\mathbf{i}}=2 \mathbf{I}
$$

where $\mathbf{I}$ is the second order identity tensor.

Next, let $\mathbf{S}$ be the unit vector defined by

$$
\mathbf{S}(\theta, \phi)=\sin \phi\left(\cos \theta \mathbf{e}_{1}+\sin \theta \mathbf{e}_{2}\right)+\cos \phi \mathbf{e}_{3}
$$

where $\theta$ is the angle measured counterclockwise about the $\mathbf{e}_{\mathbf{3}}$ from the $\mathbf{e}_{\mathbf{1}}$ direction of the projection of $\mathbf{S}$ onto the $\mathbf{e}_{\mathbf{1}}-\mathbf{e}_{2}$ plane; $\varphi$ is the angle between $\mathbf{S}$ and $\mathbf{e}_{\mathbf{3}}$ (Figure 1). From these expressions, basis functions $r_{i}(\theta, \varphi)$ can be defined by

$$
r_{i}(\theta, \phi)=\frac{3}{4 \pi} \mathbf{B}_{\mathbf{i}} \bullet \mathbf{S} \otimes \mathbf{S}, \quad i=1,2 \ldots 6
$$

where $\mathbf{A} \bullet \mathbf{B}=\operatorname{tr}\left(\mathbf{A B}^{\mathbf{T}}\right)$ denotes the scalar product between two second order tensors. Using these basis functions, it is possible to characterize a five-parameter distribution function $r\left(\theta, \varphi, w_{i}\right)$ by 


$$
r\left(\theta, \phi, w_{i}\right)=\sum_{i=1}^{6} w_{i} r_{i}(\theta, \phi)
$$

where $w_{i}$ are non-negative weighting coefficients that satisfying the restriction that

$$
\int_{0}^{\pi} \int_{0}^{2 \pi} r(\theta, \phi) \sin \phi d \theta d \phi=1
$$

provided that

$$
\sum_{i=1}^{6} w_{i}=1
$$

Also, the weighting factor in Equation 5 has been introduced so that when $w_{i}$ are all equal

$$
r\left(\theta, \phi, w_{i}\right)=\frac{1}{4 \pi}, \quad \text { for } w_{i}=\frac{1}{6}
$$

\subsection{Stress response of the fibre bundles}

Using the representation in Equation 6 of the distribution of the orientation of fibres, a simple mechanical model is proposed, which characterizes the response of the fibre bundles in the $\mathbf{N}_{\mathbf{i}}$ directions. Within the context of this model, each fibre bundle is characterised as a single elastin fibre in parallel with a distribution of undulated collagen fibres. The material fibre bundle, which was oriented in the $\mathbf{N}_{\mathbf{i}}$ direction in the reference configuration is mapped by the deformation gradient $\mathbf{F}$ to the fibre bundle, which in the present configuration has the direction $\mathbf{s}_{\mathbf{i}}$ and stretch $\lambda_{i}$, such that

$$
\begin{aligned}
\lambda_{i} \mathbf{s}_{\mathbf{i}}=\mathbf{F N}_{\mathbf{i}}, \quad \text { for } i & =1,2, \ldots 6 \text { with no sum on } i \\
\lambda_{i} & =\sqrt{\mathbf{C} \bullet \mathbf{B}_{\mathbf{i}}}
\end{aligned}
$$

where $\mathbf{C}=\mathbf{F}^{T} \mathbf{F}$ is the right-Cauchy-Green deformation tensor. Since each fibre bundle is assumed to act only in tension, it is necessary to introduce additional resistance to compression. This can be done by recalling that the dilation $J$ is a pure measure of volume change, which is defined by

$$
J=\operatorname{det}(\mathbf{F})>0
$$

Moreover, it can be shown that $J$ and $\lambda_{i}$ satisfy the evolution equations

$$
\begin{gathered}
\dot{J}=\boldsymbol{J} \mathbf{D} \mathbf{I} \\
\dot{\lambda}_{i}=\lambda_{i} \mathbf{D} \bullet\left(\mathbf{s}_{\mathbf{i}} \otimes \mathbf{s}_{\mathbf{i}}\right) \quad \text { no sum on } i
\end{gathered}
$$


where a superposed - denotes material time differentiation and $\mathbf{D}$ is the rate of deformation tensor, which is the symmetric part of the velocity gradient $\mathbf{L}$

$$
\mathbf{D}=\frac{1}{2}\left(\mathbf{L}+\mathbf{L}^{\mathbf{T}}\right)
$$

The strain energy function $W$ per unit mass of the collection of six fibre bundles is

$$
W=f(J)+\sum_{i=1}^{6} w_{i}\left(W_{e}\left(\lambda_{i}\right)+W_{c}\left(\lambda_{i}\right)\right)
$$

where $f(J)$ controls the response to compression, $W_{e}(\lambda)$ is the strain energy of the elastin fibre, and $W_{c}(\lambda)$ is the strain energy of the undulated bundle of collagen fibres. It is assumed that the properties of each fibre bundle are identical so that $W_{e}$ and $W_{c}$ have the same functional forms for each orientation.

The response of this composite material is assumed to be elastic with no mechanical dissipation so that

$$
\mathbf{T} \bullet \mathbf{D}=\rho \dot{W}
$$

where $\mathbf{T}$ is the symmetric Cauchy stress and the conservation of mass

$$
\rho J=\rho_{0}
$$

relates the present value $\rho$ of mass density to its reference value $\rho_{0}$. Using Equations 13 and 14 and the usual argument that the stress is independent of the rate of deformation, it can be shown that Equation 18 will be satisfied for all processes provided that the stress takes the form

$$
\mathbf{T}=\rho_{0} \frac{d f}{d J} \mathbf{I}+\sum_{i=1}^{6} w_{i}\left(\frac{\lambda_{i}}{J} \rho_{0}\left(\frac{d W_{e}\left(\lambda_{i}\right)}{d \lambda_{i}}+\frac{d W_{c}\left(\lambda_{i}\right)}{d \lambda_{i}}\right)\right)\left(\mathbf{s}_{\mathbf{i}} \otimes \mathbf{s}_{\mathbf{i}}\right)
$$

Moreover, $\mathbf{T}=-p \mathbf{I}+\mathbf{T}^{\prime}$, where $p$ is the pressure and $\mathbf{T}^{\prime}$ is the deviatoric part of $\mathbf{T}$ :

$$
\begin{gathered}
\mathbf{T}^{\prime}=\sum_{i=1}^{6} w_{i}\left(\frac{\lambda_{i}}{J} \rho_{0}\left(\frac{d W_{e}\left(\lambda_{i}\right)}{d \lambda_{i}}+\frac{d W_{c}\left(\lambda_{i}\right)}{d \lambda_{i}}\right)\right)\left(\mathbf{s}_{\mathbf{i}} \otimes \mathbf{s}_{\mathbf{i}}-\frac{1}{3} \mathbf{I}\right) \\
p=-\rho_{0} \frac{d f}{d J}-\frac{1}{3} \sum_{i=1}^{6} w_{i}\left(\frac{\lambda_{i}}{J} \rho_{0}\left(\frac{d W_{c}\left(\lambda_{i}\right)}{d \lambda_{i}}+\frac{d W_{e}\left(\lambda_{i}\right)}{d \lambda_{i}}\right)\right)
\end{gathered}
$$

An additional simplification can be introduced by constraining the material response to be incompressible, i.e. $J=1$. In this case, the pressure in Equation 21 is replaced by an arbitrary function of position and time that is determined by the equations of motion and boundary conditions. 
Motivated by the work of Watton et al. [26] and Gundiah et al. [27], an elastin fibre is characterised by the strain energy function

$$
\rho_{0} W_{e}(\lambda)=\frac{E_{e}}{4}\left\langle\lambda^{2}-1\right\rangle
$$

where $E_{e}$ is a material parameter representing the stiffness of the elastin fibre and the Macaulay brackets are defined by $\langle z\rangle=(z+|z|) / 2$. It follows from Equation 22 that

$$
\begin{aligned}
& \lambda \rho_{0} \frac{\partial W_{e}(\lambda)}{\partial \lambda}=\frac{1}{2} E_{e} \lambda^{2} \text { for } \lambda \geq 1, \\
& \lambda \rho_{0} \frac{\partial W_{e}(\lambda)}{\partial \lambda}=0 \text { for } \lambda<1
\end{aligned}
$$

For each $\lambda_{i}$, the total strain energy due to the elastin fibres is given by

$$
\rho_{0} W_{e}=\sum_{i=6}^{6} w_{i} \frac{E_{e}}{4}\left(\lambda_{i}^{2}-1\right)
$$

It follows that for evenly-weighted fibres $\left(w_{i}=1 / 6\right)$

$$
\rho_{0} W_{e}=\frac{E_{e}}{12}(\mathbf{C} \bullet \mathbf{I}-3)
$$

which represents an isotropic neo-Hookean material.

\subsection{Undulation distribution models of collagen fibres}

As discussed by Lanir [5], the collagen fibres are typically coiled in the stress-free reference configuration. It is assumed that a collagen fibre cannot resist compression and that its stress response is linear with extension once it has been fully straightened. In particular, the coiled nature of an individual collagen fibre is characterized by an undulation parameter $x \geq 1$, which is the value of stretch of that fibre when it first starts to support tension. Taking $D(x)$ to be the normalised distribution of the undulation

$$
\int_{1}^{\infty} D(x) d x=1
$$

the fraction of fibres that are straight at a stretch $\lambda$ is given by

$$
\int_{1}^{\lambda} D(x) d x
$$

Moreover, it is assumed that $D(x)$ vanishes for $x<1$ and that the elastic modulus $E_{c}$ of each collagen fibre is the same so the stiffness of the bundle of fibres is given by 
$E_{c} \int_{1}^{\lambda} D(x) d x$. Consequently, using Equation 20 with $J=1$, the stiffness of the collagen bundle is

$$
\frac{d}{d \lambda}\left(\lambda \rho_{0} \frac{d W_{c}(\lambda)}{d \lambda}\right)=E_{c} \int_{1}^{\lambda} D(x) d x
$$

Next, assuming that all the collagen fibres are slack and do not contribute to stress for stretches $\lambda$ less than unity, Equation 28 is integrated to give

$$
\lambda \rho_{0} \frac{d W_{c}(\lambda)}{d \lambda}=E_{c} \int_{1}^{\lambda} \int_{1}^{\lambda} D(x) d x d \lambda
$$

Thus Equations 23 and 29 can be substituted into Equations 20 and 21 to give the total stress response of the incompressible $(J=1)$ tissue model with six discrete fibre bundles.

Next, different forms for the distribution function $D(x)$ of the undulation parameter are investigated, which yield analytical expressions for the strain energy function of undulated collagen fibres.

\section{Step distribution}

$$
D(x)=\left\{\begin{array}{l}
0, \quad x<x_{1} \text { or } x>x_{3} \\
\frac{1}{x_{3}-x_{1}}, \quad \text { otherwise }
\end{array}\right.
$$

where $x_{1}$ and $x_{3}$ are described in Figure 2(a). The associated strain energy function $W_{c}$ for this distribution Equation 30 is given by

$$
\begin{aligned}
& \left.\left.\begin{array}{l}
\lambda \rho_{0} \frac{\partial W_{c}}{\partial \lambda}=0, \quad \rho_{0} W_{c}=0, \quad \lambda<x_{1} \\
\lambda \rho_{0} \frac{\partial W_{c}}{\partial \lambda}=E_{c} \frac{\left(\lambda-x_{1}\right)^{2}}{2\left(x_{3}-x_{1}\right)} \\
\left.\rho_{0} W_{c}=E_{c} \frac{1}{4\left(x_{3}-x_{1}\right)}\left[\left(\lambda-x_{1}\right)\left(\lambda-3 x_{1}\right)+2 x_{1}^{2} \ln \left(\frac{\lambda}{x_{1}}\right)\right]\right\}, \quad x_{1} \leq \lambda<x_{3} \\
\lambda \rho_{0} \frac{\partial W_{c}}{\partial \lambda}=E_{c}\left[\frac{\left(x_{3}-x_{1}\right)}{2}+\left(\lambda-x_{3}\right)\right] \\
\left.\rho_{0} W_{c}=E_{c}\left[\frac{1}{4\left(x_{3}-x_{1}\right)}\left[\left(x_{3}-x_{1}\right)\left(x_{3}-3 x_{1}\right)+2 x_{1}^{2} \ln \left(\frac{x_{3}}{x_{1}}\right)\right]+\right]\right\}, \quad \lambda>x_{3} \\
{\left[\frac{x_{3}-x_{1}}{2}-x_{3}\right] \ln \left(\frac{\lambda}{x_{3}}\right)+\left(\lambda-x_{3}\right)}
\end{array}\right]\right\}, \quad
\end{aligned}
$$


In particular, it can be seen from Equations 20 and 31 that the stress is zero until $\lambda=x_{1}$, it is then a parabolic function of $\lambda$ in the region $x_{1}<\lambda \leq x_{3}$, and, finally, it is a linear function of $\lambda$ in the region $\lambda>x_{3}$.

\section{Triangular distribution}

$$
D(x)=\left\{\begin{array}{cc}
0, \quad x<x_{1} \text { or } x>x_{3} \\
\frac{2\left(x-x_{1}\right)}{\left(x_{3}-x_{1}\right)\left(x_{2}-x_{1}\right)}, & x_{1} \leq x \leq x_{2} \\
\frac{2\left(x_{3}-x\right)}{\left(x_{3}-x_{1}\right)\left(x_{3}-x_{2}\right)}, & x_{2} \leq x \leq x_{3}
\end{array}\right.
$$

where $x_{1}, x_{2}$, and $x_{3}$ are described in Figure 2(b). Analytical expressions can also be obtained for the associated strain energy function $W_{c}$ associated with this distribution but they are not recorded here.

\section{Normal distribution}

The normal distribution function is given by

$$
D(x)=\frac{1}{\sqrt{2 \pi x_{2}^{2}}} e^{\frac{-\left(x-x_{1}\right)^{2}}{2 x_{2}^{2}}}
$$

where $x_{1}$ is the mean stretch at which the fibres are straight and $x_{2}$ is the standard deviation about the mean. This function is illustrated in Figure 2(c).

\section{$3 \quad$ Alternative constitutive models for soft tissues}

In the next sections the predictions of the proposed six-fibre model are compared with the following constitutive models of soft tissues that appear in the literature.

\section{Exponential Strain Energy Function}

A strain energy function has been used to represent the mechanical behaviour of collagen fibres of the form

$$
\rho_{o} W_{c}(\varepsilon)=x_{1}\left[\exp \left(x_{2} \varepsilon\right)-1\right]
$$

where $x_{1}$ and $x_{2}$ are material parameters and $\varepsilon=\left(\lambda^{2}-1\right) / 2$ is the fibre Green's strain. The axial Cauchy stress for the fibre is given by

$$
T=\lambda^{2} \frac{\partial W}{\partial \varepsilon}
$$


This constitutive relation has been used to model the behaviour of aortic valve cusp [16]. Equations 23 and 29 can be substituted into Equations 20 and 21 to give the total stress response of the incompressible tissue model.

\section{Langevin-based model}

An orthotropic hyperelastic model with a strain energy function of the form

$\rho_{0} W=\rho_{0} W_{0}+\frac{n k \theta}{4}\left[N \sum_{i}^{4}\left(\frac{\rho^{(i)}}{N} \beta_{\rho}^{(i)}+\ln \frac{\beta_{\rho}^{(i)}}{\sinh \beta_{\rho}^{(i)}}\right)-\frac{\beta_{P}}{P} \ln \left(\lambda_{a}^{a^{2}} \lambda_{b}^{b^{2}} \lambda_{c}^{c^{2}}\right)\right]+B\{\cosh (J-1)-1\}$

has been used to model the response of the composite tissue with fibres. The terms in the strain energy function are given in Table 1 and the associated constitutive equation for the Cauchy stress is given by

$$
\mathbf{T}=\frac{1}{J} \mathbf{F} \frac{\partial W}{\partial \mathbf{E}} \mathbf{F}^{\mathbf{T}}
$$

where $\mathbf{E}=\left(\mathbf{F}^{\mathrm{T}} \mathbf{F}-\mathbf{I}\right) / 2$ is the Lagrangian strain field. Further details of this model can be found in Bischoff et al. [28].

\section{$4 \quad$ Anisotropy of the six-fibre bundle model}

The work in Elata and Rubin [25] shows how the tensors of the six-fibre system can be used to obtain isotropic response for a strain energy function which is a quadratic function of Lagrangian strain. Since here the strain energy function is a nonlinear function of strain it is of interest to quantify the extent of possible anisotropy of the model based on only six fibres.

Consider a solid sheet of material, which is represented by the six-fibre model with equal weights for the fibres. The incompressible solid is subjected to an equibiaxial stretch in the $\mathbf{e}_{\mathbf{1}}-\mathbf{e}_{\mathbf{2}}$ plane. There is zero normal stress in the $\mathbf{e}_{3}$ direction and the deformation gradient is given by

$$
\mathbf{F}=\left(\begin{array}{ccc}
\lambda & 0 & 0 \\
0 & \lambda & 0 \\
0 & 0 & 1 / \lambda^{2}
\end{array}\right)
$$

where $\mathbf{e}_{\mathbf{i}}$ is a fixed right-handed orthonormal triad of vectors. Also, let $\mathbf{e}_{\mathbf{i}}^{\prime}$ be another right handed orthonormal triad of vectors defined by the angles $\alpha$ and $\beta$, such that 


$$
\begin{aligned}
& \mathbf{e}_{1}^{\prime}=\cos \beta \mathbf{e}_{1}+\sin \beta \mathbf{e}_{3} \\
& \mathbf{e}_{2}^{\prime}=-\sin \alpha \sin \beta \mathbf{e}_{1}+\cos \alpha \mathbf{e}_{2}+\sin \alpha \cos \beta \mathbf{e}_{3} \\
& \mathbf{e}_{3}^{\prime}=-\cos \alpha \sin \beta \mathbf{e}_{1}-\sin \alpha \mathbf{e}_{2}+\cos \alpha \cos \beta \mathbf{e}_{3}
\end{aligned}
$$

and define the rotation tensor $\mathbf{R}$ by

$$
\mathbf{R}=\mathbf{e}_{\mathbf{i}}^{\prime} \otimes \mathbf{e}_{\mathbf{i}}
$$

where the usual summation convention over repeated indices is applied. Different orientations of the material are obtained by rotating the vectors $\mathbf{N}_{\mathrm{i}}$ to the vectors $\mathbf{N}_{\mathbf{i}}^{\prime}$ defined by

$$
\mathbf{N}_{\mathbf{i}}^{\prime}=\mathbf{R N}_{\mathbf{i}}
$$

Now, let $\mathbf{T}$ be the stress tensor predicted by the model and $\mathbf{T}_{\mathrm{e}}$ be the stress tensor expected by the model, then

$$
\begin{gathered}
\mathbf{T}=T_{i j}^{\prime} \mathbf{e}_{\mathbf{i}}^{\prime} \otimes \mathbf{e}_{\mathrm{ij}}^{\prime} \\
\mathbf{T}_{\mathbf{e}}=\frac{1}{2}\left(T_{11}^{\prime}+T_{22}^{\prime}\right)\left(\mathbf{e}_{\mathbf{1}}^{\prime} \otimes \mathbf{e}_{\mathbf{1}}^{\prime}+\mathbf{e}_{\mathbf{2}}^{\prime} \otimes \mathbf{e}_{\mathbf{2}}^{\prime}\right)
\end{gathered}
$$

where use has been made of the boundary condition $T_{33}^{\prime}=0$. The magnitude of the error between the predicted and expected stress tensors is defined

$$
\text { Error }=\sqrt{\left(\mathbf{T}-\mathbf{T}_{\mathbf{e}}\right) \bullet\left(\mathbf{T}-\mathbf{T}_{\mathbf{e}}\right)}
$$

For an incompressible equally-weighted six-fibre model, the total strain energy is given by

$$
\rho_{0} W=\frac{1}{6} \sum_{i=1}^{6} f\left(\lambda_{i}\right)
$$

where $f(\lambda)$ is the strain energy function of each fibre. Using Equation 3, it can be shown that if $f(\lambda)$ is specified by

$$
f(\lambda)=3 C_{1}\left(\lambda^{2}-1\right)
$$

where $C_{1}$ is a constant, Equation 45 describes a neo-Hookean material

$$
\rho_{0} W=C_{1}(\mathbf{C} \bullet \mathbf{I}-3)
$$

Using Equation 46, the stress predicted by the model for equibiaxial stretch is isotropic and matches the expected stress response for any arbitrary rotation angles $\alpha$ and $\beta$ (Figure 3(a)).

However, for the simple case where each of the fibres has a constant stiffness $E$, $f(\lambda)$ is specified by 


$$
\begin{gathered}
f(\lambda)=E(\lambda-1-\ln (\lambda)) \\
\frac{d}{d \lambda}\left(\lambda \frac{d f}{d \lambda}\right)=E
\end{gathered}
$$

the response of Equation 45 is not isotropic for arbitrary rotation angles $\alpha$ and $\beta$ (Figure 3(b-d)). This is an undesirable effect of the description of the representation of the fibre field with a discrete number of fibres, which has been discussed previously [29].

\section{Comparison of distribution functions}

The effect of the different distribution functions was explored by specifying a parameter set for the normal distribution function and determining parameter sets for the step and triangular functions that best matched the normal distribution. The parameter sets were determined using non-linear optimisation. The lsqnonlin function in MATLAB Version 7.5 (The MathWorks, Inc., Natick, MA, USA), which uses a trust-region method, was used to minimise an objective function in a least-squares sense. The objective function used in the optimisations was

$$
F_{i}(x)=\sum_{i=1}^{n}\left(R_{i}^{\text {model }}(\mathbf{x})-R_{i}^{\text {exp }}\right)^{2}
$$

where $n$ is the number of data points, $\mathbf{x}$ is a vector representing the model parameters, $R_{i}^{\text {model }}(\mathbf{x})$ is the datum calculated from the model at the $i^{\text {th }}$ datapoint and $R_{i}^{\exp }$ is the measured 'experimental' datum at the $i^{\text {th }}$ datapoint.

The error-of-fit between the model and the synthetic experiment was calculated as follows

$$
\text { Error }- \text { of }- \text { fit }=\frac{\sum_{i=1}^{n}\left|R_{i}^{\text {model }}(\mathbf{x})-R_{i}^{\exp }\right|}{\sum_{i}^{n}\left|R_{i}^{\exp }\right|} \times 100 \%
$$

The step distribution function matched the normal distribution with an error-of-fit of $38.7 \%$ (Figure 4(a) and Table 2). The error-of-fit of the triangular distribution function was $7.7 \%$. The collagen fibre stress predicted by each model with the optimised parameter sets was also calculated (Figure 4(a)). The error-of-fit of the 

4(b)).

\section{Simulation of in vitro experiments}

The proposed model was used to simulate several uniaxial, biaxial and simple shear tests. The experimental data was taken from rabbit-skin, pig-skin, aortic valve cusps, and myocardial tissue $[1-3,30]$. The parameter values that best fit the model to experimental data were found using the same nonlinear optimisation procedures used in Section 5. The objective functions used are specified below. The error-of-fit between the model and the experiment was calculated using Equation 51.

\section{Biaxial stretching of rabbit-skin}

The model was used to fit experimental data from a biaxial test on rabbit-skin [3]. The specimen was stretched in one direction of the plane of biaxial test, the dimension in the other direction in the plane was held constant and the stretch in the third direction was determined by the incompressibility condition. The orthotropic nature of the skin was demonstrated by applying the stretch in the longitudinal direction and then the transverse direction. The width and length of the sample were both $35 \mathrm{~mm}$. Lanir and Fung [3] did not state the thickness of the sample in the paper. Here the thickness was assumed to be $1.0 \mathrm{~mm}$.

The objective function used in the optimisation was

$$
F_{i}(x)=\sum_{i=1}^{n}\left(\frac{R_{i}^{\text {model }}(\mathbf{x})-R_{i}^{\text {exp }}}{R_{i}^{\exp }}\right)^{2}
$$

All material parameters were optimised for each discrete-fibre model (Figure 5 and Table 3). The error-of-fits of the triangular and step distribution models were $8.7 \%$ and $10.2 \%$, respectively. The exponential function model simulated the data with an error-of-fit of $19 \%$. The parameters of the Bischoff et al. model were also optimised to simulate the experimental data with an error-of-fit of $15.5 \%$

\section{Uniaxial stretching of pig-skin}

Ankersen et al. [30] conducted uniaxial tensile tests on skin samples taken from the abdomen of pigs. Loads were applied along directions both parallel and perpendicular to the spine to demonstrate the orthotropic characteristics of the pig- 
skin. The elastin fibres were ignored when fitting the model to the pig-skin data. The objective function used in the optimisations was Equation 50. The optimisation procedure was the same as for the rabbit-skin optimisation (Figure 6 and Table 4). The error-of-fits of the step, triangular, and normal distribution models were less than $8.0 \%$. The exponential model did not simulate accurately the experiment with an error-of-fit was $17.3 \%$. The error-of-fit of the Bischoff et al. model was $26.6 \%$.

\section{Equibiaxial stretching of aortic valve cusp}

Billiar and Sacks [2] performed biaxial tension tests on in vitro aortic valve samples. Square samples of widths $10 \mathrm{~mm}$ to $16 \mathrm{~mm}$ were loaded quasi-statically with membrane stresses ranging from $2.5 \mathrm{Nm}^{-1}$ to $60 \mathrm{Nm}^{-1}$ applied to each side. The discrete-fibre model with the various distribution functions were fitted to the results of the $60 \mathrm{Nm}^{-1}$ equibiaxial loading of fixed and fresh samples. The reported mean thickness of fresh samples was $0.466 \mathrm{~mm}$ and the mean thickness of the fixed samples was $0.457 \mathrm{~mm}$. The objective function in Equation 52 was used in the non-linear optimisation. In this case, the stretches predicted by the models were compared with the experimental stretches.

All the model parameters were optimised to simulate the fresh aortic valve cusp experimental data (Figure 7(a) and Table 5) and the fixed experimental data (Figure 7(b) and Table 6). The Bischoff et al. model was also optimised to simulate the experimental data with an error-of-fit of $2.1 \%$ for the fresh cusps and $2.3 \%$ for the fixed cusps.

\section{Simple shear of rat septal myocardium}

Dokos et al. [1] determined the stress response of rat septal myocardium samples subjected to simple shear deformations. The deformations were applied in two mutually orthogonal anatomical directions on the top surface of the samples - the posterior-anterior direction (PA) and apex-base direction (AB). The samples measured $2.5 \mathrm{~mm} \times 2.5 \mathrm{~mm} \times 5 \mathrm{~mm}$. The myocardium exhibited a non-linear, anisotropic, and viscoelastic stress-strain response. When fitting the models to the experimental data, the viscoelastic characteristics were ignored and only the loading part of each curve was utilised. For the material parameter optimisation, the elastin fibres were ignored and $\theta$ (defined in Figure 1) was also optimised in addition to the 
other parameters. The objective function in Equation 52 was used in the non-linear optimisation.

The error-of-fits were $13.3 \%$ for the normal distribution model, $9.3 \%$ for the triangular distribution model, and 9.1\% for the step distribution model (Figure 8 and Table 7). The error-of-fit for the exponential function was $8.6 \%$. The parameters of the Bischoff et al. model were also optimised to simulate the experimental data with an error-of-fit of $13.8 \%$

\section{Parameter study}

The sensitivity of the material parameters was investigated by increasing and decreasing each parameter of the triangular function model by $10 \%$ of its optimised value from the rabbit-skin simulations (Table 3). The error-of-fit and residual was most sensitive to changes in the parameters of the triangular distribution function $-x_{1}$, $x_{2}$, and $x_{3}$ (Table 8 and Figure 9). Decreasing $x_{1}, x_{2}$, and $x_{3}$ resulted in the skin 'locking' at lower stretches. Changes to the collagen and elastin stiffnesses and fibreweights had little effect on the model behaviour.

\section{Discussion}

Accurate constitutive models of fibrous soft tissue have many applications including the prediction of the behaviour of arterial tissue during aging and disease [26], and the development of surgical simulators [31]. It is a goal of any constitutive model to be as simple as possible while still capturing the salient features of the soft tissue it is simulating, such as non-linearity and anisotropy.

A simple model for the response of fibrous soft tissues has been proposed using six discrete-fibre bundles. The overall strain energy is the weighted sum of the strain energy of each fibre bundle. A novel aspect of the model is the use of simple analytical distribution functions to represent the undulation of bundles of collagen fibres. This approach yields a closed form analytical expression for the strain energy function for bundle of collagen fibres that avoids the sometimes costly numerical integration of some statistical distribution functions. The model parameters are all physically-based including two directly representing the stiffness of the collagen and elastin fibres and five independent weights representing the orientation of the fibres within the tissue. Depending on the distribution function used, two or three parameters determine the stretch at which the collagen fibres are engaged. 
The triangular distribution function can match the normal distribution function with greater accuracy than the step distribution function (Figure 4(a) and Table 2). However, the collagen fibre stress predicted using the different distribution functions are very similar (Figure 4(b)). This would suggest that the two-parameter step function is sufficient for modelling the uncrimping of collagen fibres.

The discrete-fibre model accurately simulated the deformation of several different tissue types under different loading protocols. In general, there was little difference in the goodness-of-fit of the model to the experimental data whether the two-parameter step distribution function or the three-parameter triangular distribution function was used. In particular, for the biaxial stretching of rabbit-skin, the error-of-fit of the triangular function model $(8.7 \%)$ was only slightly better than the error-of-fit of the step function model $(10.2 \%)$.

The analytical distribution function models performed as well as the normal distribution model. In fact, for the rat septal myocardium simulation, the error-of-fit of the normal distribution model $(13.3 \%)$ was higher than the error-of-fit of the analytical distribution models $(9.1 \%$ and $9.3 \%)$.

Modelling the uncrimping of the collagen fibres with an exponential function did not result in accurate simulation of the pig and rabbit-skin stretch tests (Table 2 and Table 4). The exponential function model did accurately simulate the simple shear of myocardium and biaxial loading of aortic cusp samples. It is also noted that Billiar and Sacks [16] achieved good fits for aortic valve cusp data using the same exponential function along with a Gaussian distribution function representing the orientation of the collagen fibres. Nevertheless, the results of the exponential model suggest that the mechanical behaviour of the collagen fibres is not adequately represented, in general, by an exponential function within the proposed discrete-fibre model.

The Bischoff et al. model [28] simulated the biaxial stretching of rabbit-skin (error-of-fit of 15.5\%), fresh and fixed aortic cusp (error-of-fit 2.1\% and 2.3\%), and rat septal myocardium (error-of-fit 13.8\%). However, its ability to simulate the uniaxial stretching of pig-skin was relatively poor (Table 4). Also, a limitation of the Bischoff et al. model is that the inverse Langevin function is undefined when its argument is greater than unity. This leads to difficulties in calculations of mechanical behaviour when in the 'locking' region at large stretch. This can be circumvented somewhat by using an inexact series representation of the inverse Langevin function. 
However, this does not capture the high non-linearity observed in soft tissues at large stretches Bischoff et al. [32].

In addition to the quality of the fits of the analytical distribution functions, the solution times of the step distribution model were about one-third of the solution times of the normal distribution model (in Matlab R2007 on Windows XP Professional Intel Core ${ }^{\mathrm{TM}}, 2.6 \mathrm{GHz}, 3.5 \mathrm{~GB}$ RAM). While this reduction in time is dependent on the solution methods used, it does indicate potential savings when solving large finite element models of soft tissue mechanics. These results further indicate that, for several soft-tissue test cases, the two-parameter step distribution function is sufficient to model the uncrimping of collagen fibres under load.

The predicted stiffness of the collagen fibres range from about $8 \mathrm{MPa}$ for the rat septal myocardial tissue to $900 \mathrm{MPa}$ for the pig-skin. The predicted elastin fibre stiffness ranged from about $2 \mathrm{kPa}$ for the rabbit-skin to $150 \mathrm{kPa}$ for the fixed aortic valve cusp. While the upper bounds of these stiffness ranges are similar to values reported in the literature, the lower bounds are less than reported values. Collagen fibre stiffnesses have been estimated in different tissue types to range from about 100 $\mathrm{MPa}$ to as high as $4.4 \mathrm{GPa}[7,18,33]$. The reported stiffness of elastin fibres range from $100 \mathrm{kPa}$ to about $1 \mathrm{MPa}[18,26,34]$. The parameter study of the triangular distribution function has shown that the error-of-fit is least sensitive to elastin and collagen fibre stiffness and most dependent on when the collagen fibres uncrimp. Indeed, for the pig-skin and septal myocardium simulations the elastin fibres were ignored. This does not mean that the elastin fibre contribution to the mechanical response of the soft tissue is negligible. It just indicates that from a modelling perspective its presence did not improve the fit in these particular cases.

Uniqueness of an optimised set of parameters may be an issue for the proposed soft tissue model with ten independent material parameters. Since all the model parameters have a physical meaning, the issue can be minimised by choosing values based on physical measurements. For example, the fibre-weights can be determined from imaging and measuring the fibre distributions within the soft tissue [35]. In addition, the soft tissue should be subject to a rich set of deformations in order to characterise its complex constitutive behaviour and thus improve the identifiability of the parameters [36, 37].

The proposed discrete-fibre model is not polyconvex. A polyconvex constitutive equation improves material stability and guarantees the existence of a deformation 
state that minimises the elastic strain energy [13]. This is an important condition when solving boundary value problems [15]. An effort was made to guarantee polyconvexity by defining the discrete-fibre model in terms of generalised stretch invariants in a manner similar to that considered in [38]. However, the resulting model was unsuccessful in matching the experimental data.

The anisotropy of the model detailed in Section 4 is a result of representing a fibre field with a discrete number of fibres and using a general nonlinear strain energy function based on the distribution of fibre undulation. The anisotropy induced by numerical integration over the unit sphere has been investigated for other constitutive models $[29,39,40]$. While the anisotropy is an undesirable effect, the maximum error in the test cases is about 5\%, which is probably less than the experimental error in many soft tissue deformation experiments.

Viscoelasticity, a characteristic common to all soft tissues, has been ignored in the proposed model. This will be incorporated into future developments. Finally, it will be of interest to incorporate the proposed model within a finite element framework to test its ability to simulate more complex boundary value problems.

\section{Acknowledgements}

MB Rubin would like to thank the Auckland Bioengineering Institute for hosting him during part of his sabbatical leave from Technion. The authors would also like to acknowledge useful discussions with AE Ehret, M Itskov, M Nash and H Schmid. 


\section{References}

1. Dokos S, LeGrice IJ, Smaill BH, Kar J, Young AA. A triaxial-measurement shear-test device for soft biological tissues. Journal of Biomechanical Engineering 2000; 122(5):471-78.

2. Billiar KL, Sacks MS. Biaxial mechanical properties of the natural and glutaraldehyde treated aortic valve cusp - Part I: Experimental results. Journal of Biomechanical Engineering 2000; 122(1):2330.

3. Lanir Y, Fung YC. Two-dimensional mechanical properties of rabbit skin--II. Experimental results. Journal of Biomechanics 1974; 7(2):171-74.

4. Tong P, Fung Y-C. The stress-strain relationship for the skin. Journal of Biomechanics 1976; 9(10):649-57.

5. Lanir Y. Constitutive equations for fibrous connective tissues. Journal of Biomechanics 1983; 16(1):1-12.

6. Bischoff JE, Arruda EM, Grosh K. Finite element modeling of human skin using an isotropic, nonlinear elastic constitutive model. Journal of Biomechanics 2000; 33(6):645-52.

7. Lokshin O, Lanir Y. Micro and macro rheology of planar tissues. Biomaterials 2009; 30(17):311827.

8. Capelli C, Taylor AM, Migliavacca F, Bonhoeffer P, Schievano S. Patient-specific reconstructed anatomies and computer simulations are fundamental for selecting medical device treatment: Application to a new percutaneous pulmonary valve. Philosophical Transactions of the Royal Society A: Mathematical, Physical and Engineering Sciences 2010; 368(1921):3027-38.

9. Lott-Crumpler DA, Chaudhry HR. Optimal patterns for suturing wounds of complex shapes to foster healing. Journal of Biomechanics 2001; 34(1):51-58.

10. Hendriks FM, Brokken D, Oomens CWJ, Bader DL, Baaijens FPT. The relative contributions of different skin layers to the mechanical behavior of human skin in vivo using suction experiments. Medical Engineering and Physics 2006; 28(3):259-66.

11. Karwoski AC, Plaut RH. Experiments on peeling adhesive tapes from human forearms. Skin Research and Technology 2004; 10(4):271-77.

12. Silver FH, Kato YP, Ohno M, Wasserman AJ. Analysis of mammalian connective tissue: Relationship between hierarchical structures and mechanical properties. Journal of Long-Term Effects of Medical Implants 1992; 2(2-3):165-98.

13. Kroon M, Holzapfel GA. A new constitutive model for multi-layered collagenous tissues. Journal of Biomechanics 2008; 41(12):2766-71.

14. Itskov M, Ehret AE, Mavrilas D. A polyconvex anisotropic strain-energy function for soft collagenous tissues. Biomechanics and Modeling in Mechanobiology 2006; 5(1):17-26.

15. Itskov M, Ehret AE. A universal model for the elastic, inelastic and active behaviour of soft biological tissues. GAMM Mitteilungen 2009; 32(2):221-36.

16. Billiar KL, Sacks MS. Biaxial mechanical properties of the native and glutaraldehyde-treated aortic valve cusp: Part II - A structural constitutive model. Journal of Biomechanical Engineering 2000; 122(4):327-36.

17. Zulliger MA, Fridez P, Hayashi K, Stergiopulos N. A strain energy function for arteries accounting for wall composition and structure. Journal of Biomechanics 2004; 37(7):989-1000.

18. Wuyts FL, Vanhuyse VJ, Langewouters GJ, Decraemer WF, Raman ER, Buyle S. Elastic properties of human aortas in relation to age and atherosclerosis: A structural model. Physics in Medicine and Biology 1995; 40(10):1577-97.

19. Raghupathy R, Barocas VH. A closed-form structural model of planar fibrous tissue mechanics. Journal of Biomechanics 2009; 42(10):1424-28.

20. Freed AD, Einstein DR, Vesely I. Invariant formulation for dispersed transverse isotropy in aortic heart valves: An efficient means for modeling fiber splay. Biomechanics and Modeling in Mechanobiology 2005; 4(2-3):100-17. 
21. Gasser TC, Ogden RW, Holzapfel GA. Hyperelastic modelling of arterial layers with distributed collagen fibre orientations. Journal of the Royal Society Interface 2006; 3(6):15-35.

22. Wilkes GL, Brown IA, Wildnauer RH. The biomechanical properties of skin. CRC critical reviews in bioengineering 1973; 1(4):453-95.

23. Holzapfel GA, Gasser TC, Ogden RW. A new constitutive framework for arterial wall mechanics and a comparative study of material models. Journal of Elasticity 2000; 61(1-3):1-48.

24. Caner FC, Carol I. Microplane constitutive model and computational framework for blood vessel tissue. Journal of Biomechanical Engineering 2006; 128(3):419-27.

25. Elata D, Rubin MB. Isotropy of strain energy functions which depend only on a finite number of directional strain measures. Journal of Applied Mechanics, Transactions ASME 1994; 61(2):28489.

26. Watton PN, Ventikos Y, Holzapfel GA. Modelling the mechanical response of elastin for arterial tissue. Journal of Biomechanics 2009; 42(9):1320-25.

27. Gundiah N, B Ratcliffe M, A Pruitt L. Determination of strain energy function for arterial elastin: Experiments using histology and mechanical tests. Journal of Biomechanics 2007; 40(3):586-94.

28. Bischoff JE, Arruda EA, Grosh K. A microstructurally based orthotropic hyperelastic constitutive law. Journal of Applied Mechanics, Transactions ASME 2002; 69(5):570-79.

29. Ehret AE, Itskov M, Schmid H. Numerical integration on the sphere and its effect on the material symmetry of constitutive equations - A comparative study. International Journal for Numerical Methods in Engineering 2010; 81(2):189-206.

30. Ankersen J, Birkbeck A, Thomson R, Vanezis P. Puncture resistance and tensile strength of skin simulants. Proceedings of the Institution of Mechanical Engineers, Part H: Journal of Engineering in Medicine 1999; 213(6):493-501.

31. Bro-Nielsen M. Finite element modeling in surgery simulation. Proceedings of the IEEE 1998; 86(3):490-503.

32. Bischoff JE, Arruda EM, Grosh K. Finite element simulations of orthotropic hyperelasticity. Finite Elements in Analysis and Design 2002; 38(10):983-98.

33. Silver FH, Freeman JW, DeVore D. Viscoelastic properties of human skin and processed dermis. Skin Research and Technology 2001; 7(1):18-23.

34. Lillie MA, Gosline JM. Mechanical properties of elastin along the thoracic aorta in the pig. Journal of Biomechanics 2007; 40(10):2214-21.

35. Noorlander ML, Melis P, Jonker A, Van Noorden CJF. A quantitative method to determine the orientation of collagen fibers in the dermis. Journal of Histochemistry and Cytochemistry 2002; 50(11):1469-74.

36. Flynn C, Taberner A, Nielsen P. Mechanical characterisation of in vivo human skin using a 3D force-sensitive micro-robot and finite element analysis. Biomechanics and Modeling in Mechanobiology. In Press. doi:10.1007/s10237-010-0216-8

37. Lanir Y, Lichtenstein O, Imanuel O. Optimal design of biaxial tests for structural material characterization of flat tissues. Journal of Biomechanical Engineering 1996; 118(1):41-46.

38. Ehret AE, Itskov M, Weinhold G. A Viscoelastic Anisotropic Model for Soft Collageneous Tissues Based on Distributed Fiber-Matrix Units. In: Garikipati K, Arruda EM, eds. IUTAM Symposium on Cellular, Molecular and Tissue Mechanics. Springer Netherlands, 2010: 55-65.

39. Bazant ZP. Efficient Numerical integration on the surface of a sphere. Zeitschrift fur angewandte Mathematik und Mechanik 1986; 66(1):37-49.

40. Itskov M, Ehret AE, Dargazany R. A full-network rubber elasticity model based on analytical integration. Mathematics and Mechanics of Solids. doi:10.1177/1081286509106441 


\section{Figure Legends}

Figure 1. Discrete six-fibre model: each fibre is parallel to the lines that pass through opposing vertices of a regular icosahedron

Figure 2. Distribution functions for the undulation of collagen fibres: (a) Step; (b) Triangular; (c) Normal

Figure 3. Equibiaxial stretching of a soft tissue sheet in the 1-2 plane with the fibre field rotated $\alpha$ degrees about the $\mathbf{e}_{1}$ axis and $\beta$ degrees about the $\mathbf{e}_{2}$ axis. The fibres are equally weighted. In (a) the strain energy function for each fibre has a neo-Hookean form (Equation 46) and the predicted response of the model is identical to the expected for isotropic response. In (b), (c) and (d), the strain energy function is Equation 48. When there is a rotation of the fibre field, the predicted response of the model is anisotropic and deviates from the expected response.

Figure 4. (a) Comparison of goodness-of-fits of the step and triangular distribution models when simulating the distribution curve generated by the normal distribution model. The parameters for each model are given in Table 2. (b) The corresponding collagen fibre stress for each distribution model.

Figure 5. Simulation of biaxial stretching of rabbit-skin using different distribution functions for the undulation of collagen fibres, including comparison with exponential and Bischoff et al. [28] models. The model parameters are recorded in Table 3. Bischoff et al. model parameters are $n=4.4830 \times 10^{22}$, $a=1.889, b=1.351, c=1.096, B=50 \mathrm{kPa}$.

Figure 6. Simulation of uniaxial stretching of pig-skin using different distribution functions for the undulation of collagen fibres, including comparison with exponential and Bischoff et al. [28] models. The model parameters are recorded in Table 4. Bischoff et al. model parameters are $n=3.9698 \times 10^{24}$, $a=1.383, b=0.632, c=1.302, B=100 \mathrm{kPa}$.

Figure 7. Simulation of equibiaxial tension loading of (a) fresh and (b) fixed aortic valve cusps using different distribution functions for the undulation of collagen fibres, including comparison with exponential and Bischoff et al. [28] models. The model parameters are recorded in Table 5 and Table 6. Bischoff et al. model parameters are $n=6.0 \times 10^{17}, a=2.05, b=1.70, c=0.865, B=100 \mathrm{kPa}$ for the fresh sample, and $n=7.0 \times 10^{17}, a=1.85, b=1.35, c=0.822, B=500 \mathrm{kPa}$ for the fixed sample.

Figure 8. Simulation of simple shear of rat myocardium using different distribution functions for the undulation of collagen fibres, including comparison with exponential and Bischoff et al. [28] models. (a) Posterior-anterior direction; (b) Apex-base direction. The model parameters are recorded in Table 7. Bischoff et al. model parameters are $n=3.569 \times 10^{23}, a=1.403, b=1.479, c=1.509, B=100 \mathrm{kPa}$.

Figure 9. Effect of changing model parameters by $10 \%$ of their optimised values for the rabbit-skin simulations based on the parameters of the triangular distribution function recorded in Table 3 
Table 1. Definition of parameters in the Bischoff et al. strain energy function [28]

\begin{tabular}{|c|c|}
\hline$W_{0}$ & constant related to the strain energy of undeformed fibres \\
\hline$n$ & Number of fibres per unit volume of the network \\
\hline$k$ & Boltzman's constant, $1.38 \times 10^{-23} \mathrm{JK}^{-1}$ \\
\hline$\theta$ & Absolute temperature \\
\hline$N$ & Number of freely jointed rigid links \\
\hline$\beta_{\rho}^{(i)}=L^{-1}\left(\rho^{(i)} / N\right)$ & Inverse Langevin function where $L(x)=\operatorname{coth} x-1 / x$ \\
\hline$P$ & $\begin{array}{l}\text { Undeformed fibre length; } P=\sqrt{a^{2}+b^{2}+c^{2}} / 2 ; a, b \text {, and } c \text { are } \\
\text { the orthotropic cell dimensions. }\end{array}$ \\
\hline $\begin{array}{l}\lambda_{a}=\sqrt{\mathbf{a}^{\mathrm{T}} \mathbf{C a}} \\
\lambda_{b}=\sqrt{\mathbf{b}^{\mathrm{T}} \mathbf{C b}} \\
\lambda_{c}=\sqrt{\mathbf{b}^{\mathrm{T}} \mathbf{C c}}\end{array}$ & $\begin{array}{l}\text { Principal stretches along the principal material axes for } \\
\text { network. } \mathbf{C}=\mathbf{F}^{T} \mathbf{F} \text { is the right Cauchy stress tensor. } \mathbf{a}, \mathbf{b} \text {, and } \\
\mathbf{c} \text { form the axes that describe the orientation of the orthotropic } \\
\text { cell. }\end{array}$ \\
\hline$B$ & Bulk modulus \\
\hline$J$ & Volume ratio; $J=\operatorname{det} \mathbf{F}$ \\
\hline
\end{tabular}


Table 2. Optimised parameters for the step and triangular distribution functions when simulating the synthetic distribution curve generated by the normal distribution model and exhibited in Figure 4(a).

\begin{tabular}{|l|l|l|l|l|l|}
\hline & $x_{\mathbf{1}}$ & $\boldsymbol{x}_{\mathbf{2}}$ & $\boldsymbol{x}_{\mathbf{3}}$ & Residual & Error (\%) \\
\hline Normal & 1.200 & 0.075 & - & - & - \\
\hline Step & 1.345 & - & 1.670 & 38.97 & 38.7 \\
\hline Triangular & 1.273 & 1.498 & 1.732 & 1.287 & 7.7 \\
\hline
\end{tabular}

\begin{tabular}{|c|c|c|c|c|c|c|c|c|}
\hline & $\begin{array}{l}E_{c} \\
(\mathrm{MPa})\end{array}$ & $\begin{array}{l}E_{e} \\
(\mathbf{k P a})\end{array}$ & $x_{1}$ & $x_{2}$ & $x_{3}$ & $\left(w_{1}, w_{2}, w_{3}, w_{4}, w_{5}, w_{6}\right)$ & Residual & $\begin{array}{l}\text { Error } \\
(\%)\end{array}$ \\
\hline Step & 103.2 & 2.06 & 1.224 & - & 4.174 & $\begin{array}{l}0.0125,0.0007,0.4639,0.4 \\
639,0.0007,0.0584\end{array}$ & 2.3 & 10.2 \\
\hline Triangular & 117.45 & 2.31 & 1.217 & 1.413 & 2.034 & $\begin{array}{l}0.0062,0.00018,0.45757 \\
0.45757,0.000143,0.078\end{array}$ & 2.4 & 8.7 \\
\hline Normal & 69.2 & 2.464 & 1.350 & 0.044 & - & $\begin{array}{l}0.0020,0.0001,0.4534,0.4 \\
534,0.0001,0.0912\end{array}$ & 2.6 & 8.6 \\
\hline Exponential & - & 1.610 & 0.038 & 9.240 & - & $\begin{array}{l}0.0080,0.0000,0.4778,0.4 \\
778,0.0000,0.0364\end{array}$ & 4.3 & 19.0 \\
\hline $\begin{array}{l}\text { Bischoff et } \\
\text { al. [28] }\end{array}$ & - & - & - & - & - & - & 2.4 & 15.5 \\
\hline
\end{tabular}

Table 4. Optimised material parameters for modelling the uniaxial stretching of pig-skin exhibited in Figure 6.

Note that elastin fibres are ignored in this case. Bischoff et al. model parameters are given in Figure 6.

\begin{tabular}{|c|c|c|c|c|c|c|c|}
\hline & $E_{c}(\mathrm{MPa})$ & $x_{1}$ & $x_{2}$ & $x_{3}$ & $\left(w_{1}, w_{2}, w_{3}, w_{4}, w_{5}, w_{6}\right)$ & Residual & $\begin{array}{l}\text { Error } \\
(\%)\end{array}$ \\
\hline Step & 918.5 & 1.005 & 1.074 & - & $\begin{array}{l}0.1388,0.000,0.079 \\
9,0.0799,0.000,0.6 \\
967\end{array}$ & 2.3 & 7.7 \\
\hline Triangular & 928.1 & 1.000 & 1.020 & 1.100 & $\begin{array}{l}0.1381,0.000,0.079 \\
5,0.0795,0.000,0.6 \\
965\end{array}$ & 2.3 & 7.6 \\
\hline Normal & 823.7 & 1.032 & 0.012 & - & $\begin{array}{l}0.1368,0.000,0.083 \\
5,0.0835,0.000,0.6 \\
965\end{array}$ & 2.3 & 8.0 \\
\hline Exponential & - & 16.15 & 2.24 & - & $\begin{array}{l}0.0102,0.000,0.260 \\
9,0.2609,0.000,0.4 \\
6809\end{array}$ & 9.8 & 17.3 \\
\hline
\end{tabular}




$-$

$\mid-$

\begin{tabular}{l|l|l|l}
- & - & - & - \\
\hline
\end{tabular}

\begin{tabular}{|l|l|}
\hline- & 394.2
\end{tabular}

Table 5. Optimised material parameters for modelling the equibiaxial loading of fresh aortic valve cusps exhibited in Figure 7(a). Bischoff et al. model parameters are given in Figure 7(a).

\begin{tabular}{|c|c|c|c|c|c|c|c|c|}
\hline & $\begin{array}{l}E_{c} \\
(\mathbf{M P a})\end{array}$ & $\begin{array}{l}E_{e} \\
(\mathbf{k P a})\end{array}$ & $x_{1}$ & $x_{2}$ & $x_{3}$ & $\left(w_{1}, w_{2}, w_{3}, w_{4}, w_{5}, w_{6}\right)$ & Residual & $\begin{array}{l}\text { Error } \\
(\%)\end{array}$ \\
\hline Step & 602.4 & 51.8 & 1.244 & - & 2.249 & $\begin{array}{l}0.2018,0.0007,0.1319,0 . \\
1319,0.0007,0.5329\end{array}$ & 0.053 & 2.1 \\
\hline Triangular & 604.7 & 51.6 & 1.202 & 1.468 & 2.191 & $\begin{array}{l}0.0559,0.0006,0.2195,0 . \\
2195,0.0006,0.5040\end{array}$ & 0.050 & 2.0 \\
\hline Normal & 705.2 & 43.7 & 1.455 & 0.077 & - & $\begin{array}{l}0.1161,0.0003,0.1832,0 . \\
1832,0.0003,0.5170\end{array}$ & 0.043 & 1.4 \\
\hline Exponential & - & $\begin{array}{l}29.03 \\
1\end{array}$ & 905.6 & 8.757 & - & $\begin{array}{l}0.0007,0.0001,0.1418,0 . \\
1418,0.0001,0.7153\end{array}$ & 0.053 & 1.7 \\
\hline $\begin{array}{l}\text { Bischoff et al. } \\
{[28]}\end{array}$ & - & - & - & - & - & - & 0.115 & 2.1 \\
\hline
\end{tabular}

Table 6. Optimised material parameters for modelling the equibiaxial loading of fixed aortic valve cusps exhibited in Figure 7(b). Bischoff et al. model parameters are given in Figure 7(b).

\begin{tabular}{|c|c|c|c|c|c|c|c|c|}
\hline & $\begin{array}{l}E_{c} \\
(\mathrm{MPa})\end{array}$ & $\begin{array}{l}E_{e} \\
(\mathbf{k P a})\end{array}$ & $x_{1}$ & $x_{2}$ & $x_{3}$ & $\left(w_{1}, w_{2}, w_{3}, w_{4}, w_{5}, w_{6}\right)$ & Residual & $\begin{array}{l}\text { Error } \\
(\%)\end{array}$ \\
\hline Step & 409.0 & 154.4 & 1.115 & - & 2.313 & $\begin{array}{l}0.0282,0.0000,0.2939,0 . \\
2939,0.0000,0.3840\end{array}$ & 0.017 & 0.9 \\
\hline Triangular & 364.3 & 108.7 & 1.092 & 1.185 & 2.313 & $\begin{array}{l}0.0147,0.0000,0.3089,0 . \\
3089,0.0000,0.3675\end{array}$ & 0.016 & 0.8 \\
\hline Normal & 585.0 & 174.5 & 1.175 & 0.014 & - & $\begin{array}{l}0.0924,0.00002,0.25466 \\
, 0.25466,0.00002,0.398\end{array}$ & 0.024 & 1.5 \\
\hline Exponential & - & 57.1 & 782.0 & 17.596 & - & $\begin{array}{l}0.0102,0.0000,0.2609,0 . \\
2609,0.0000,0.4681\end{array}$ & 0.016 & 0.8 \\
\hline $\begin{array}{l}\text { Bischoff et al. } \\
{[28]}\end{array}$ & - & - & - & - & - & - & 0.080 & 2.3 \\
\hline
\end{tabular}

Table 7. Optimised material parameters for modelling simple shear of rat myocardium exhibited in Figure 8. Note that elastin fibres are ignored in this case. Bischoff et al. model parameters are given in Figure 8.

\begin{tabular}{|l|l|l|l|l|l|l|l|l|}
\hline & $\begin{array}{l}E_{c} \\
(M P a)\end{array}$ & $x_{1}$ & $x_{2}$ & $x_{3}$ & $\left(w_{1}, w_{2}, w_{3}, w_{4}, w_{5}, w_{\mathbf{6}}\right)$ & $\theta\left({ }^{\mathbf{0}}\right)$ & Residual & $\begin{array}{l}\text { Error } \\
(\%)\end{array}$ \\
\hline Step & 9.187 & 1.000 & - & 6.159 & $\begin{array}{l}0.0356,0.1394,0.1161, \\
0.1808,0.1360,0.3921\end{array}$ & 3.5 & 1.333 & 9.1 \\
\hline Triangular & 8.004 & 1.000 & 1.003 & 7.117 & $0.0721,0.1094,0.0697$, & 9.3 & 1.373 & 9.3 \\
\hline
\end{tabular}




\begin{tabular}{|c|c|c|c|c|c|c|c|c|}
\hline & & & & & $0.1222,0.1009,0.5255$ & & & \\
\hline Normal & 8.094 & 1.704 & 0.544 & - & $\begin{array}{l}0.0634,0.1305,0.1209 \\
0.1088,0.0002,0.5762\end{array}$ & $\begin{array}{l}- \\
12.4\end{array}$ & 2.993 & 13.3 \\
\hline Exponential & - & 71.22 & 1.077 & - & $\begin{array}{l}0.1992,0.1976,0.0202, \\
0.0164,0.0873,0.4793\end{array}$ & 6.4 & 1.361 & 8.6 \\
\hline $\begin{array}{l}\text { Bischoff et al. } \\
{[28]}\end{array}$ & - & - & - & - & - & - & 3.428 & 13.8 \\
\hline
\end{tabular}


Table 8. Rabbit-skin error-of-fit and residuals when model parameters are each increased and decreased by $10 \%$ of their optimised values.

\begin{tabular}{|c|c|c|c|c|c|c|c|c|c|c|c|c|c|}
\hline & Optimised & \multicolumn{2}{|l|}{$E_{c}$} & \multicolumn{2}{|l|}{$E_{e}$} & \multicolumn{2}{|l|}{$x_{1}$} & \multicolumn{2}{|l|}{$x_{2}$} & \multicolumn{2}{|l|}{$x_{3}$} & \multicolumn{2}{|l|}{$w_{1}$} \\
\hline \% Change & - & -10 & +10 & -10 & +10 & -10 & +10 & -10 & +10 & -10 & +10 & -10 & +10 \\
\hline Error (\%) & 8.7 & 10.3 & 10.1 & 8.8 & 8.6 & 1942 & 77.9 & 162.2 & 38.1 & 25.2 & 21.1 & 9.8 & 8.2 \\
\hline Residual & 2.4 & 2.6 & 2.7 & 2.5 & 2.5 & 35793 & 27.7 & 117.8 & 8.2 & 6.0 & 3.9 & 2.5 & 2.5 \\
\hline
\end{tabular}




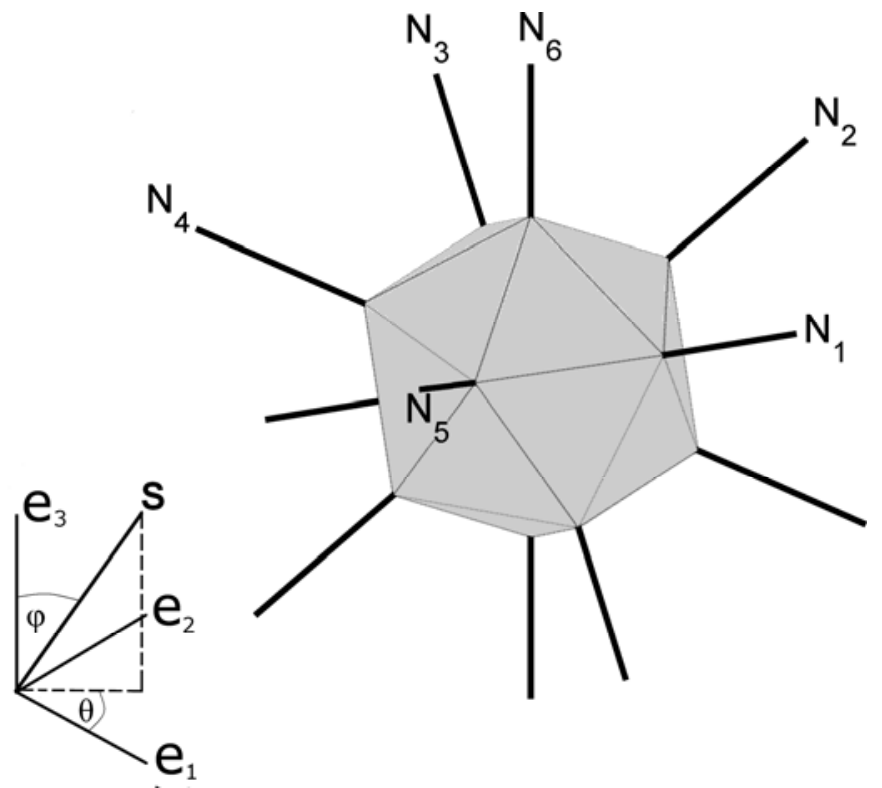

Figure 1. 


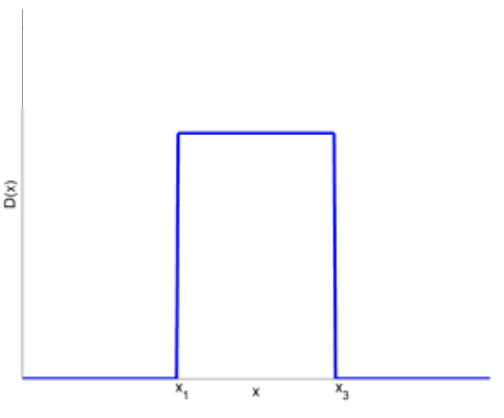

(a)

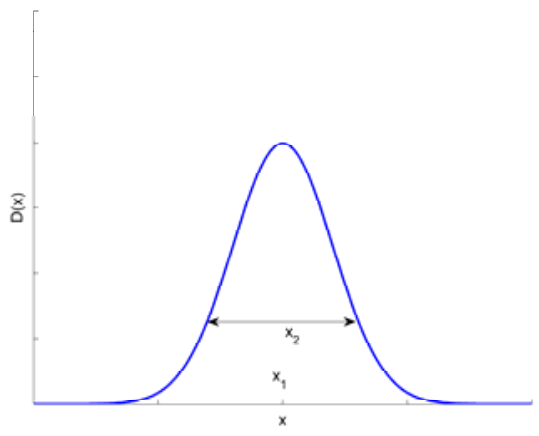

(c)

Figure 2.

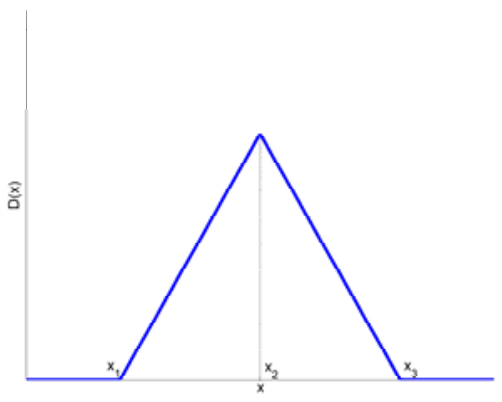

(b)

28 
(a)
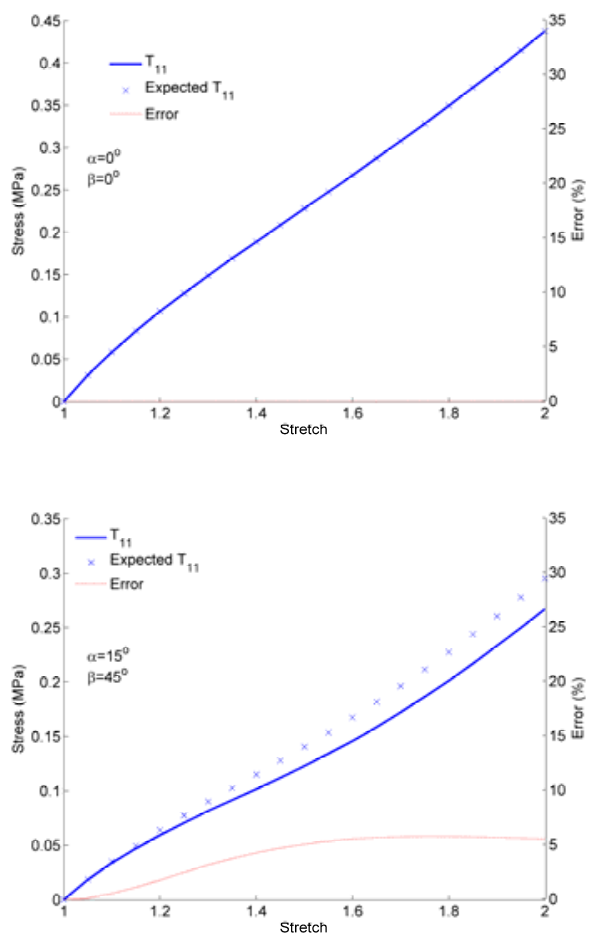

(c)

Figure 3. (b)

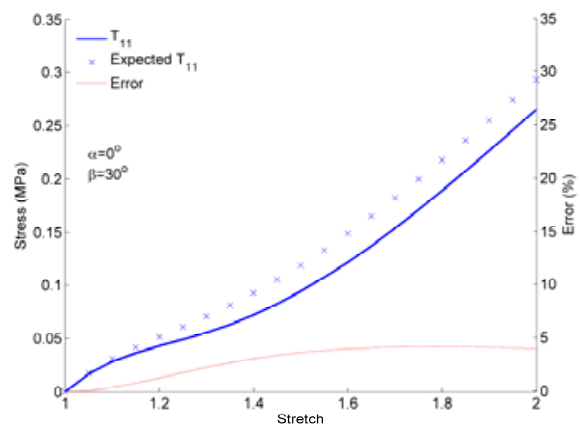

(d)

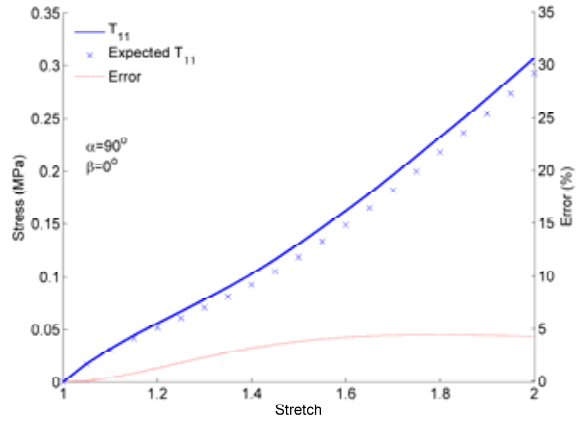




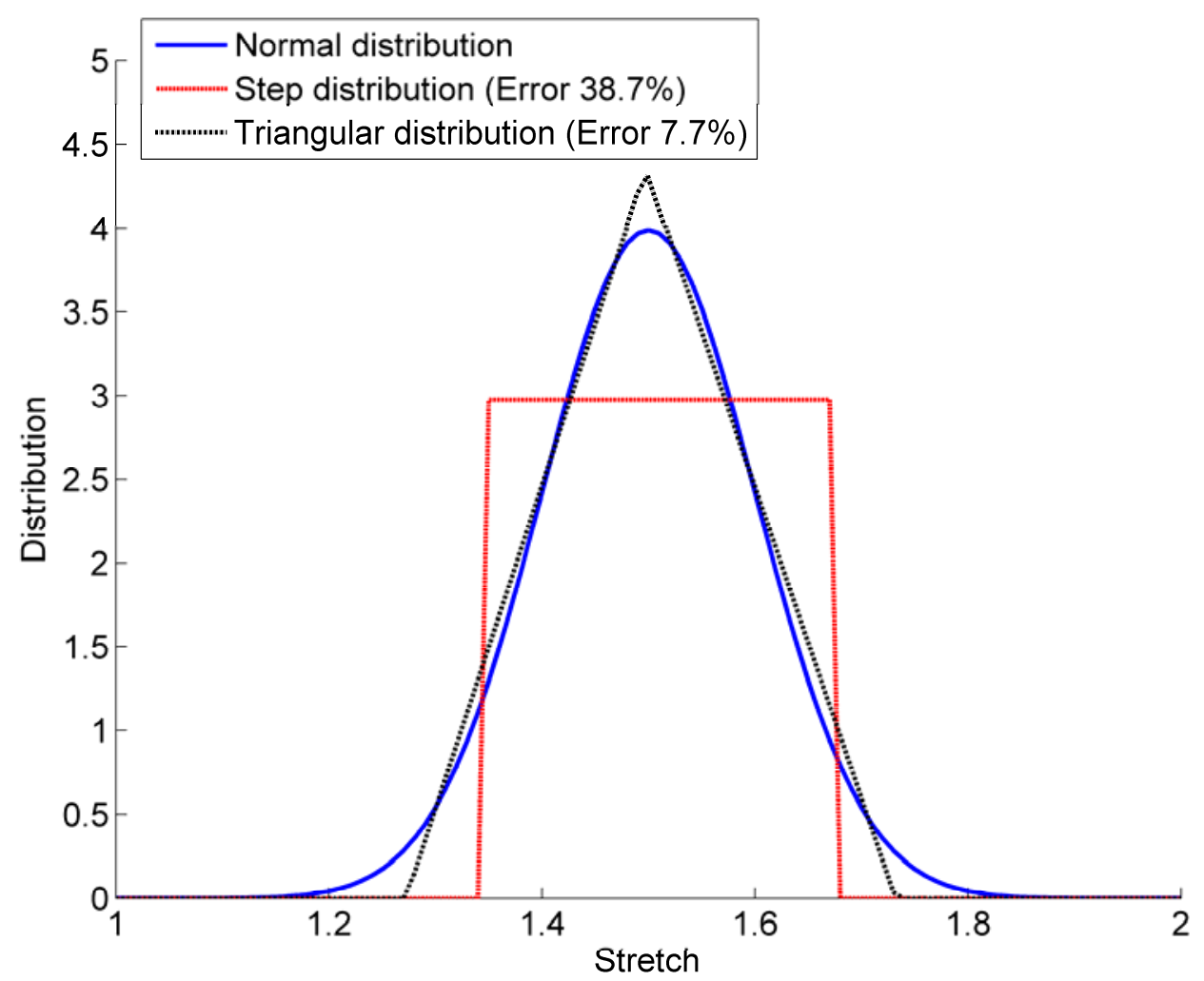

Figure 4(a).

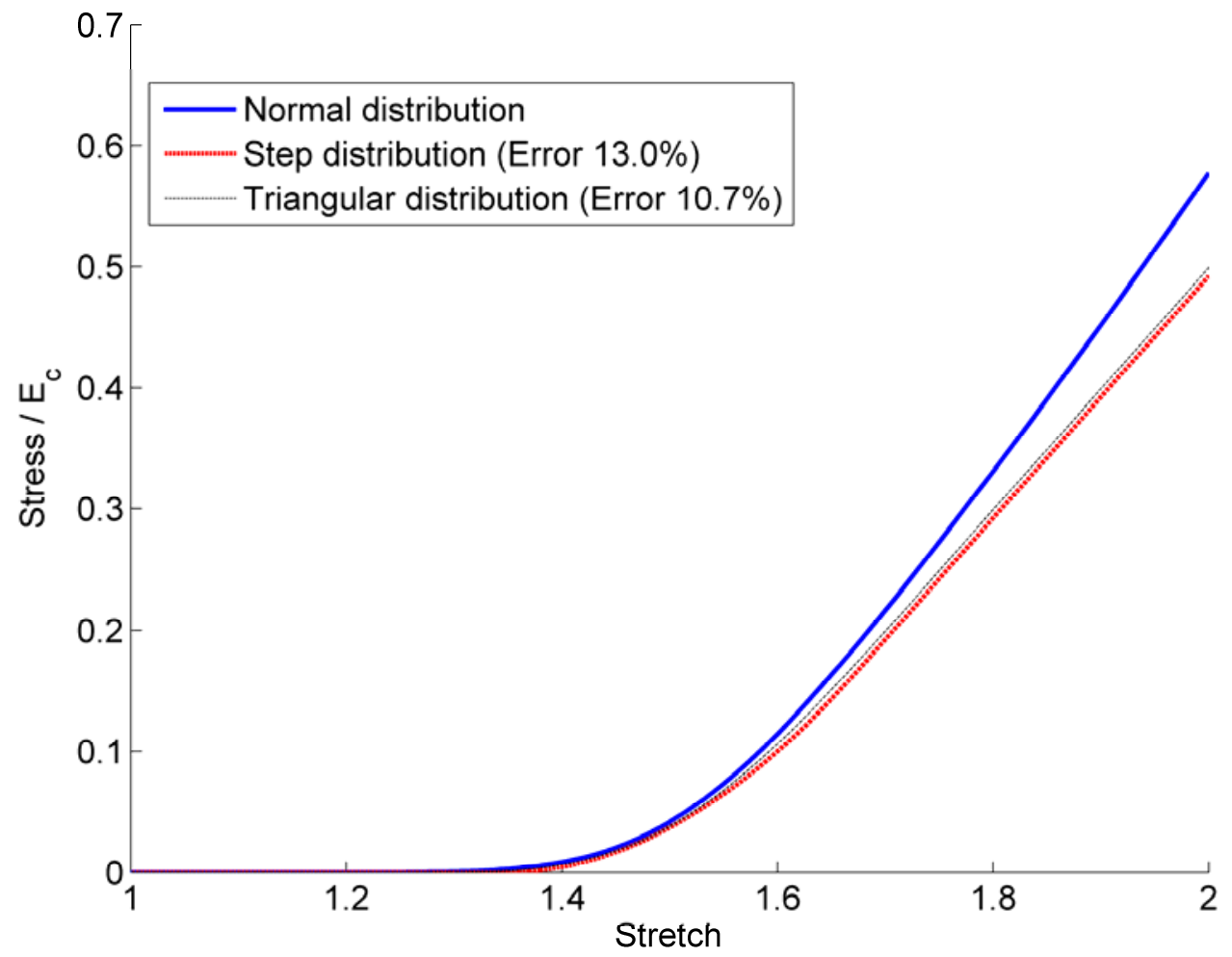

Figure 4(b). 


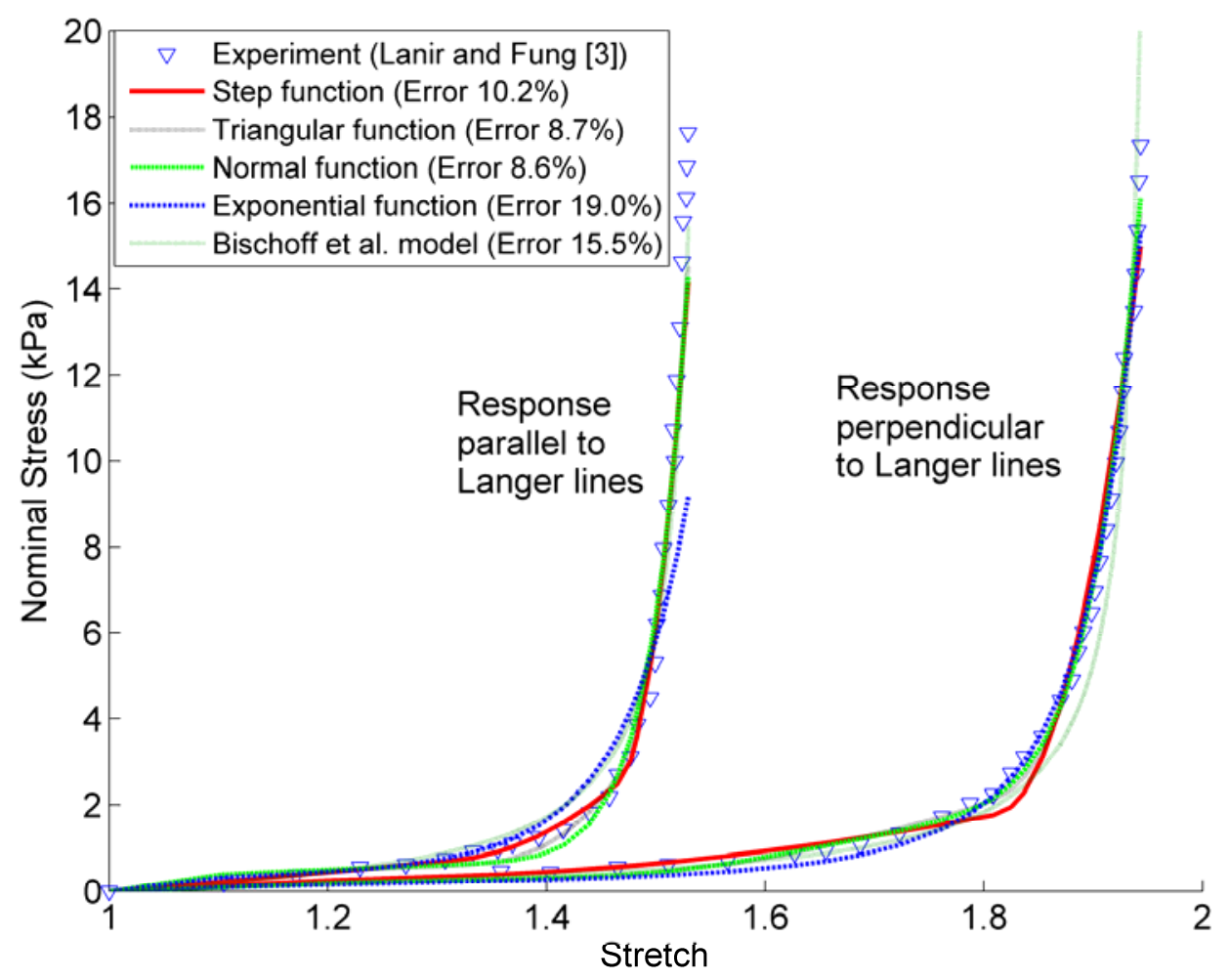

Figure 5.

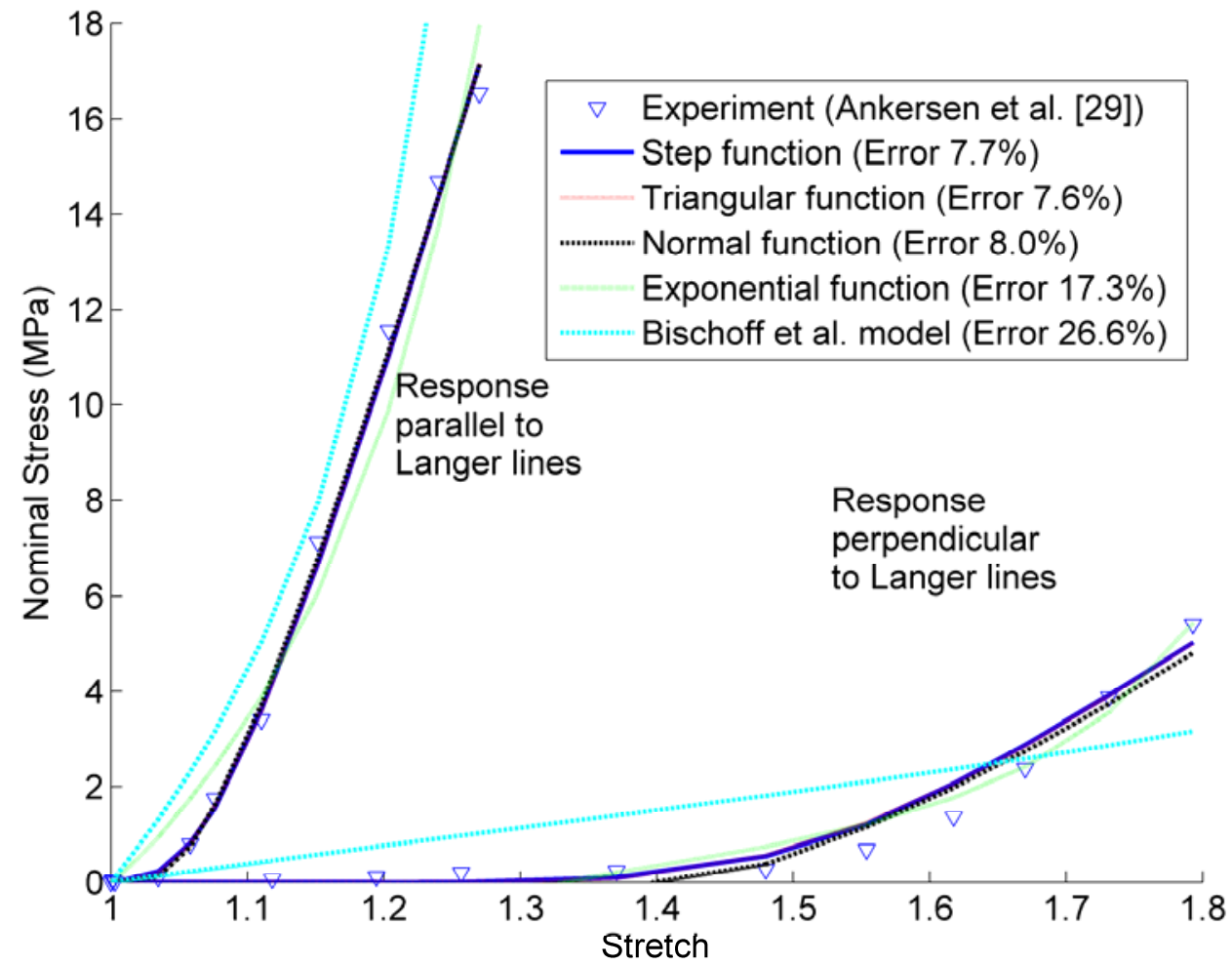

Figure 6. 


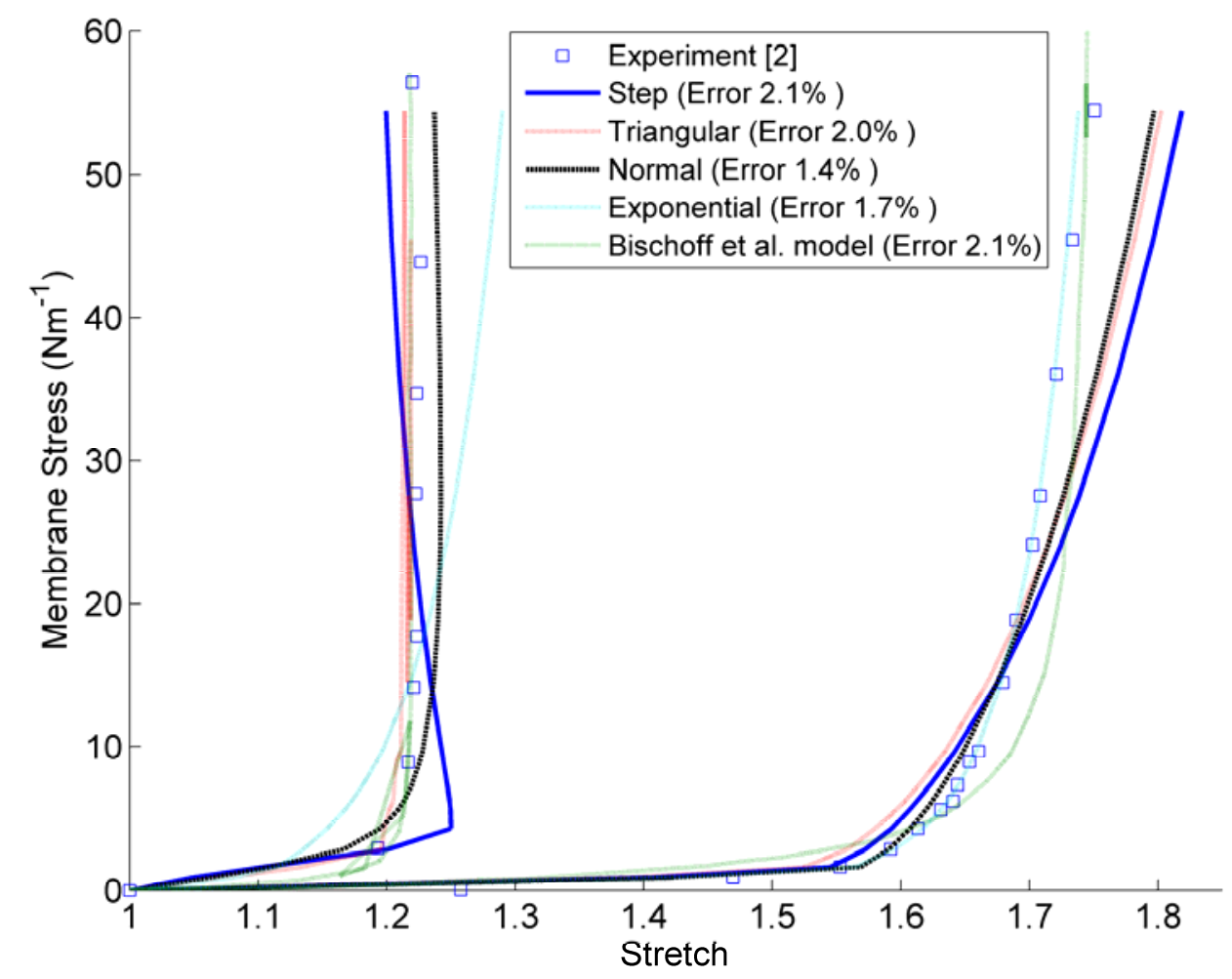

Figure 7(a).

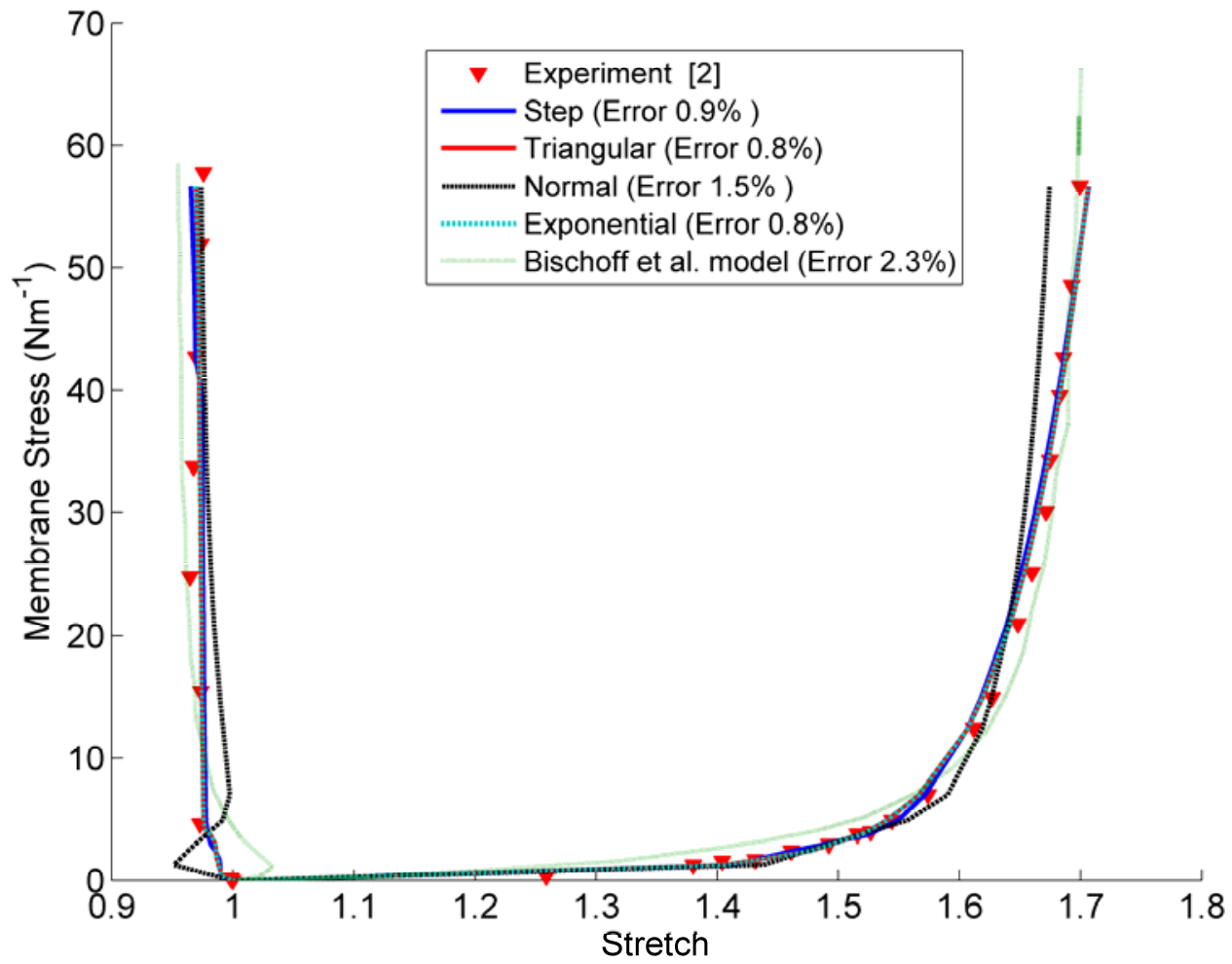

Figure 7(b). 


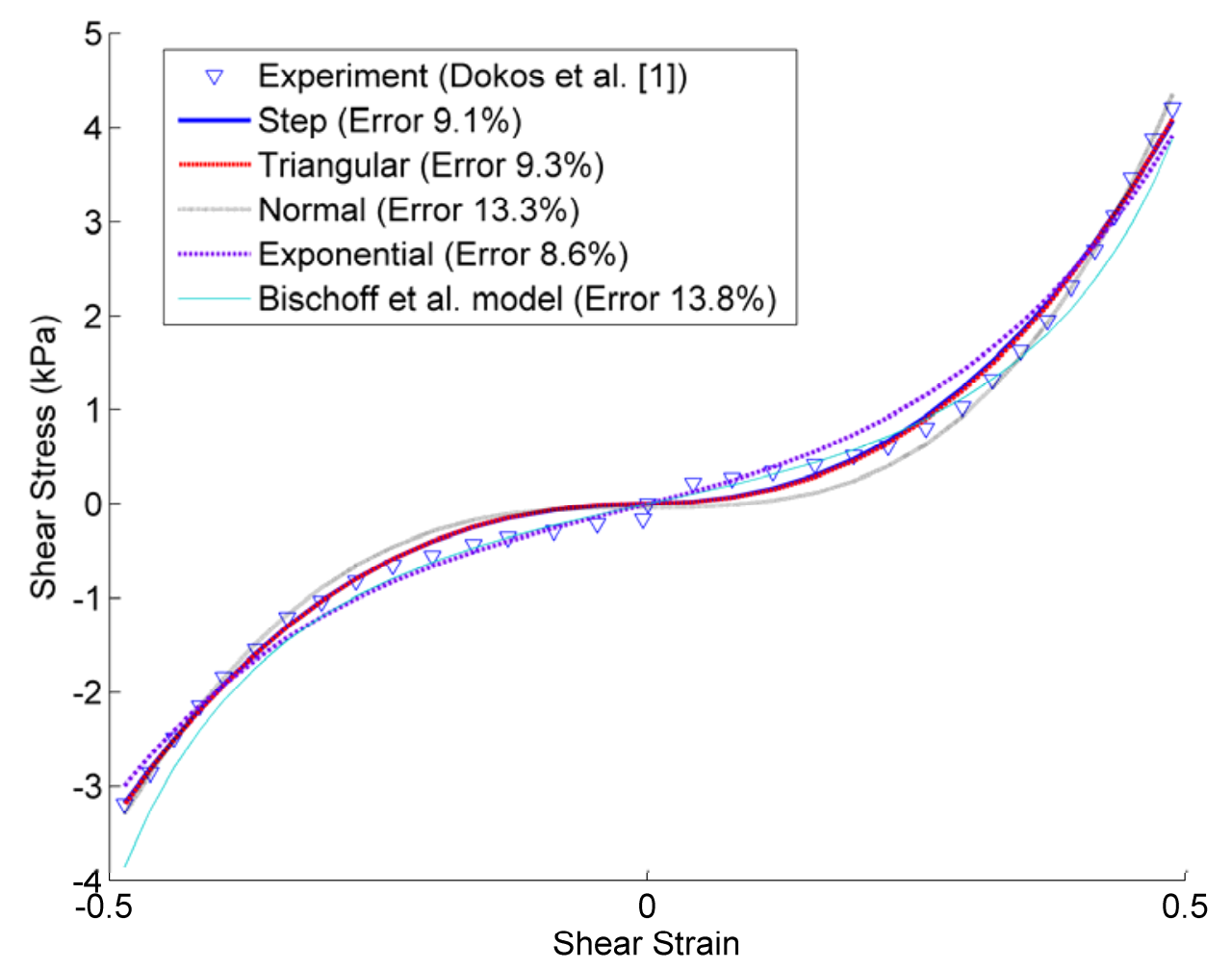

Figure 8(a).

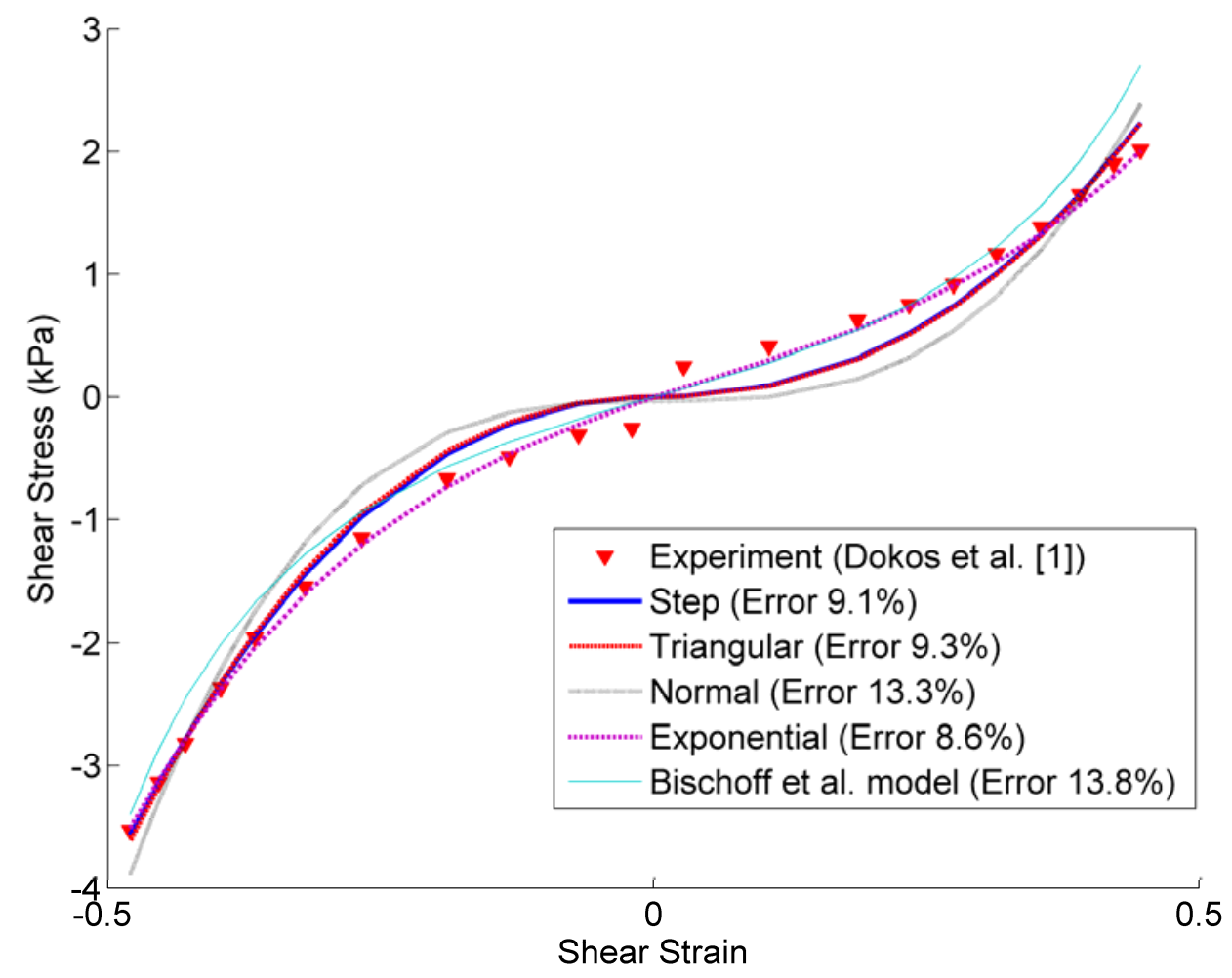

Figure $8(b)$. 
(a)

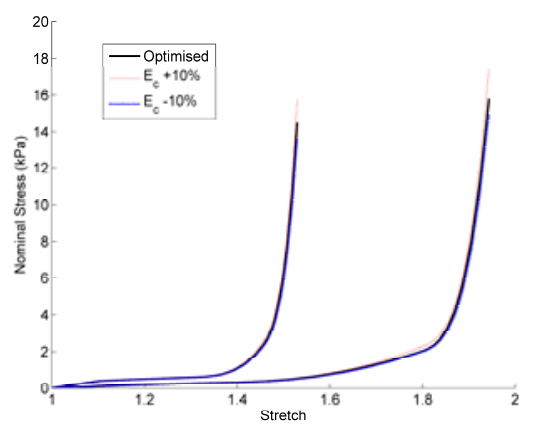

(c)

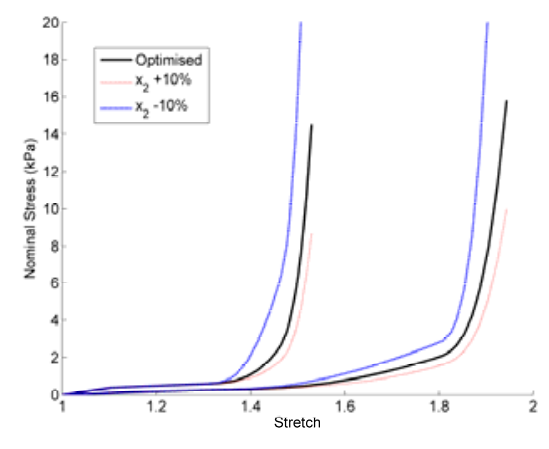

(b)

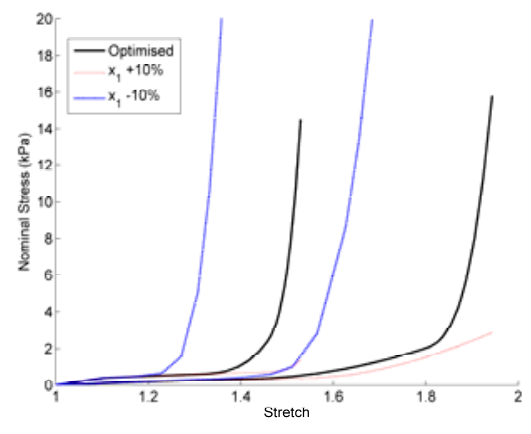

(d)

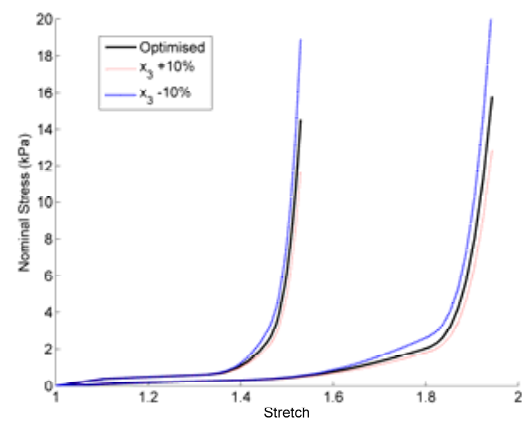

Figure 9. 



\section{RECENT REPORTS}

27/10 On Liquid Films on an Inclined Plate

Benilov

Chapman

McLoed

Ockendon

Zubkov

28/10 An a posteriori error analysis of a mixed finite element Galerkin

Memon approximation to second order linear parabolic problems

Nataraj

Pani

29/10 A Priori Error Estimates for Semidiscrete Finite Element Approx-

Goswami imations to Equations of Motion Arising in Oldroyd Fluids of Or-

Pani der One

30/10 The Landau-de Gennes theory of nematic liquid crystals: Uniaxiality versus Biaxiality

31/10 The Radial-Hedgehog Solution in Landau-de Gennes' theory

Majumdar

32/10 Nonlinear instability in flagellar dynamics: a novel modulation mechanism in sperm migration?

Gadelha

Gaffney

Smith

Kirkman-Brown

33/10 Error bounds on block GaussSeidel solutions of coupled multiphysics problem

Whiteley

Gillow

Tavener

Walter

34/10 A random projection method for sharp phase boundaries in lattice Boltzmann simulations

Reis

Dellar

35/10 Regularized Particle Filter with Langevin Resampling Step

Duan

Farmer

Moroz

36/10 Sequential Inverse Problems Bayesian Principles and the Logistic Map Example

Duan

Farmer

Moroz

37/10 Circumferential buckling instability of a growing cylindrical tube Moulton

Goriely

38/10 Preconditioners for state constrained optimal control problems

Stoll with Moreau-Yosida penalty function

Wathen

39/10 Local synaptic signaling enhances the stochastic transport of motor-driven cargo in neurons

Newby

Bressloff

40/10 Convection and Heat Transfer in Layered Sloping Warm-Water Aquifer

McKibbin

Hale

Style

Walters

41/10 Optimal Error Estimates of a Mixed Finite Element Method for

Goswami

Parabolic Integro-Differential Equations with Non Smooth Initial

Pani Data

Yadav 
42/10 On the Linear Stability of the Fifth-Order WENO Discretization

Motamed

Macdonald

Ruuth

43/10 Four Bugs on a Rectangle

Chapman

Lottes

Trefethen

44/10 Mud peeling and horizontal crack formation in drying clay

Style

Peppin

Cocks

45/10 Binocular Rivalry in a Competitive Neural Network with Synaptic Depression

Kilpatrick

Bressloff

46/10 A theory for the alignment of cortical feature maps during devel-

Bressloff opment

Oster

47/10 All-at-Once Solution if Time-Dependent PDE-Constrained Optimisation Problmes

Stoll

Wathen

48/10 Possible role of differential growth in airway wall remodeling in

Moulton asthma

Goriely

49/10 Variational Data Assimilation Using Targetted Random Walks

Cotter

Dashti

Robinson

Stuart

Copies of these, and any other OCCAM reports can be obtained from:

Oxford Centre for Collaborative Applied Mathematics Mathematical Institute

24 - 29 St Giles'

Oxford

OX1 3LB

England

www.maths.ox.ac.uk/occam 